Pacific

Journal of

Mathematics

\title{
ON CROSSED HOMOMORPHISMS OF \\ THE VOLUME PRESERVING DIFFEOMORPHISM GROUPS
}

RYOJI KASAGAWA 


\title{
ON CROSSED HOMOMORPHISMS OF THE VOLUME PRESERVING DIFFEOMORPHISM GROUPS
}

\author{
RYOJI KASAGAWA
}

\begin{abstract}
We construct crossed homomorphisms of the groups of volume preserving diffeomorphisms of closed manifolds with nontrivial first cohomology groups and give their applications to the volume flux groups. Moreover, we see that they descend to crossed homomorphisms of their isotopy groups. In the two dimensional case, we show that their restrictions to Torelli groups are the first Johnson homomorphisms.
\end{abstract}

\section{Introduction}

In this paper we construct crossed homomorphisms $J$ of the group $\mathscr{D}_{\mathrm{vol}}$ of volume preserving diffeomorphisms of a closed oriented smooth manifold $M$ of dimension $n$ with volume form vol. Each $J$ is related with a Pontryagin class $p$ of degree $k$ and takes values in $\mathscr{H}=\operatorname{Hom}\left(\bigwedge^{n-4 k} H^{1}(M ; \mathbb{R}), H^{n-1}(M ; \mathbb{R})\right)$. This construction is an analogy of that for the symplectomorphism groups of symplectic manifolds in [Kasagawa 2008]. In it, crossed homomorphisms are constructed from certain relations of Chern classes and the cohomology class of the symplectic form. In this volume case, we use relations such as $p(M) \cup a_{1} \cup \cdots \cup a_{n-4 k}=\kappa$ [vol], where $a_{i} \in H^{1}(M ; \mathbb{R})$ and $\kappa \in \mathbb{R}$. But there are usually many such relations, and the domains of crossed homomorphisms constructed from such relations need to be restricted to certain subgroups, so we consider them all together. This is the reason why the target of $J$ is the space of homomorphisms between cohomology groups as above. The crossed homomorphism $J$, which is a 1-cocycle in terms of group cohomology theory, depends on the choice of the ingredients used in the construction, but we can show that its cohomology class does only on the Pontryagin class $p$, not on the other ingredients. Some cohomology classes on groups of volume preserving diffeomorphisms were studied by McDuff [1983], but they are defined only on the identity component. A significant point of our construction is that $J$ 's and their cohomology classes are defined on the whole group of volume preserving diffeomorphisms.

MSC2010: primary 57R50, 58D05; secondary 22E65, 57R15.

Keywords: crossed homomorphisms, volume preserving diffeomorphisms, volume flux groups. 
We calculate the derivative of $J$ along smooth curves in $\mathscr{D}_{\mathrm{vol}}$. Its formula contains the derivative of the volume flux homomorphism of $(M$, vol) as a term, so we have an application of our crossed homomorphisms to the volume flux ones. As a corollary of it, we obtain some conditions for which the volume flux groups vanish, some of which have been obtained by Kędra, Kotschick, and Morita [2006]. They studied more properties of flux groups not only for the volume case, but also for the symplectic case and others. In the two dimensional case, Kotschick and Morita also studied cohomology classes of the symplectomorphism groups of surfaces related with the extensions of the flux homomorphisms in [Kotschick and Morita 2005; 2007]. Their work suggests some applications of the crossed homomorphisms $J$.

The derivative formula for $J$ tells us that the image of the identity component of $\mathscr{D}_{\mathrm{vol}}$ under $J$ is easily understood. It turns out that $J$ descends to a crossed homomorphism $\mathscr{E}$, from $\pi_{0} \mathscr{D}_{\mathrm{vol}}$ to a quotient of $\mathscr{H}$. It can be considered a crossed homomorphism of the group $\pi_{0} \mathscr{D}$ of path components of the diffeomorphism group $\mathscr{D}$ of the oriented manifold $M$ since it is isomorphic to $\pi_{0} \mathscr{D}_{\mathrm{vol}}$ by the induced homomorphism of the standard inclusion $\mathscr{D}_{\mathrm{vol}} \hookrightarrow \mathscr{D}$ by Moser [1965]. The first nontrivial example of $\mathscr{g}$ is the two dimensional case. Let $M$ be a closed oriented surface $\Sigma_{g}$ of genus $g \geqq 3$. The group $\pi_{0} \mathscr{D}$ is called the mapping class group of $\Sigma_{g}$. The standard action of it on $H_{\mathbb{Z}}=H_{1}\left(\Sigma_{g} ; \mathbb{Z}\right)$ gives the well-known representation $\pi_{0} \mathscr{D} \rightarrow \operatorname{Sp}(2 g ; \mathbb{Z})$, whose kernel $\Phi_{g}$ is called the Torelli group. Johnson [1980] defined a surjective homomorphism $\tau_{g}: \Phi_{g} \rightarrow \bigwedge^{3} H_{\mathbb{Z}} / H_{\mathbb{Z}}$, which is called the first Johnson homomorphism. If we take $p=1$ as a Pontryagin class of degree 0 , the target of the crossed homomorphism $\mathscr{g}$ is $\mathscr{H} / \sim=\operatorname{Hom}\left(\bigwedge^{2} H^{1}\left(\Sigma_{g} ; \mathbb{R}\right), H^{1}\left(\Sigma_{g} ; \mathbb{R}\right)\right) / \sim$. Using the Poincaré duality, we have a natural injection $\wedge^{3} H_{\mathbb{Z}} \rightarrow \mathcal{H}$. We can see that it induces an injective homomorphism from the target of $\tau_{g}$ to that of $\mathscr{g}$. Thus, we can compare $\tau_{g}$ with $\mathscr{F}$ on the Torelli group $\mathscr{I}_{g}$ and can show that $\mathscr{F}$ coincides with $-\tau_{g} / 2$ on $\mathscr{I}_{g}$. In other words, $\mathscr{g}$ is an extension of $-\tau_{g} / 2$ as a crossed homomorphism of the whole mapping class group with larger target. Thus, the $\mathscr{F}$ 's in dimension greater than 2 are considered analogues of the first Johnson homomorphisms for higher dimensions. On the other hand, Morita [1993] had already extended $\tau_{g}$ to a crossed homomorphism of $\pi_{0} \mathscr{D}$ with target $\frac{1}{2} \wedge^{3} H_{\mathbb{Z}} / H_{\mathbb{Z}}$. The target of Morita's crossed homomorphism is also contained in that of $\mathscr{F}$ in the same way as above. We do not know if they are essentially the same, but in this paper we don't pursue this problem.

We note that there are many ways of extending Johnson homomorphisms, such as [Day 2007; Morita 1993; Morita and Penner 2008; Perron 2004] and others, and also that some relations between first Johnson homomorphisms and flux homomorphisms in the two dimensional case were obtained [Day 2011]. So, if we consider high dimensional analogues of these works, our crossed homomorphisms give ingredients for them. 
This paper is organized as follows: In Section 2, we define the crossed homomorphisms $J$ and $\mathscr{F}$ and state the main results. In Sections 3 and 4, we show the existence of main ingredients in the construction of $J$ and some lemmas related to them. In Section 5, we recall properties of Bott homomorphisms. In Section 6, we prove Theorem 2.3, which states that the crossed homomorphism $J$ is well defined and defines a unique cohomology class. In Section 7, we calculate the derivative of $J$ along a smooth path in $\mathscr{D}_{\mathrm{vol}}$ and show some results for the volume flux homomorphisms and groups. In Section 8, we see that $J$ descends to the crossed homomorphism $\mathscr{F}$. In Section 9, we recall the first Johnson homomorphism $\tau_{g}$ of $\Sigma_{g}$. In Sections 10, 11, and 12, we explicitly describe ingredients needed to calculate $\mathscr{F}$ in the 2 dimensional case. In Sections 13 and 14 we compute $\mathscr{F}$, which shows that it coincides with $-\frac{1}{2} \tau_{g}$ on the Torelli group $\Phi_{g}$.

\section{Definitions and main results}

Let $M$ be a closed oriented smooth manifold of dimension $n$ with a volume form vol. Let $\mathscr{D}=\operatorname{Diff}_{+}(M)$ be the group of orientation preserving diffeomorphisms of $M$ and $\mathscr{D}_{\mathrm{vol}}=\left\{\varphi \in \mathscr{D} \mid \varphi^{*} \mathrm{vol}=\mathrm{vol}\right\}$ its subgroup of volume preserving ones. Put $C_{0}^{\infty}(M)=\left\{g \in C^{\infty}(M) \mid \int_{M} g\right.$ vol $\left.=0\right\}, \mathscr{D}_{\mathrm{vol}}$ then acts on it by $\varphi_{*} g=\left(\varphi^{-1}\right)^{*} g$ for $\varphi \in \mathscr{D}_{\mathrm{vol}}$ and $g \in C_{0}^{\infty}(M)$. Put $\varphi_{\sharp} h(a)=\varphi_{*}\left(h\left(\varphi^{*} a\right)\right)$ for $a \in H^{1}(M)$ and $h \in \operatorname{Hom}\left(H^{1}(M), C_{0}^{\infty}(M)\right), \mathscr{D}_{\mathrm{vol}}$ then acts on $\operatorname{Hom}\left(H^{1}(M), C_{0}^{\infty}(M)\right)$ by $(\varphi, h) \mapsto$ $\varphi_{\sharp} h$. Here, $H^{1}(M)$ is the first cohomology group of $M$ with real coefficients, and hereafter we use cohomology groups with real coefficients if not mentioned explicitly. In this paper, actions of $\mathscr{D}_{\mathrm{vol}}$ on similar spaces of homomorphisms, such as $\operatorname{Hom}\left(\wedge^{l} H^{1}(M), V\right),\left(V=\Omega^{n-1}(M), H^{n-1}(M), \ldots\right)$, are given by the same formula, $\varphi_{\sharp} g(a)=\varphi_{*}\left(g\left(\varphi^{*} a\right)\right)$, for $g$ considered there. Take a linear section $r: H^{1}(M) \rightarrow Z^{1}(M)$ of the projection $Z^{1}(M) \rightarrow H^{1}(M)$, where $Z^{1}(M)$ is the space of closed 1-forms on $M$.

Lemma 2.1. There exists a unique crossed homomorphism

$$
f: \mathscr{D}_{\mathrm{vol}} \ni \varphi \mapsto f_{\varphi} \in \operatorname{Hom}\left(H^{1}(M), C_{0}^{\infty}(M)\right)
$$

such that $d f_{\varphi}(a)=\varphi_{\sharp} r(a)-r(a)$ for each $\varphi \in \mathscr{D}_{\mathrm{vol}}$ and $a \in H^{1}(M)$.

Here, $\varphi_{\sharp} r(a)=\varphi_{*}\left\{r\left(\varphi^{*} a\right)\right\}$ as mentioned above, and by definition, $f$ is a crossed homomorphism if and only if the equality $f_{\varphi \psi}=f_{\varphi}+\varphi_{\sharp} f_{\psi}$ holds for all $\varphi, \psi \in \mathscr{D}_{\mathrm{vol}}$.

For any $a=a_{1} \wedge \cdots \wedge a_{h} \in \wedge^{h} H^{1}(M)$, put $\wedge_{r} a=r\left(a_{1}\right) \wedge \cdots \wedge r\left(a_{h}\right)$ and $\cup a=a_{1} \cup \cdots \cup a_{h}$, we then have the homomorphisms

$$
\bigwedge_{r}: \bigwedge^{h} H^{1}(M) \rightarrow \Omega^{h}(M) \text { and } \bigcup: \bigwedge^{h} H^{1}(M) \rightarrow H^{h}(M)
$$

by linear extension for each $h$. We use the same symbols $\wedge_{r}$ and $\bigcup$ for different $h$ 's. 
Let $I_{n}^{2 k}$ be the set of invariant, symmetric, multilinear functions on $\operatorname{gl}(n, \mathbb{R})$ of degree $2 k$ with values in $\mathbb{R}$. Take $p \in I_{n}^{2 k}$. We have the Pontryagin class $p(M) \in H^{4 k}(M)$ of $M$ corresponding to it. Let $A$ be a $\operatorname{GL}(n, \mathbb{R})$-connection on $T M$, and $F_{A} \in \Omega^{2}(M, \operatorname{End}(T M))$ its curvature form. We then have $p(M)=[\Delta(A) p] \in$ $H^{4 k}(M)$ by Chern-Weil theory, where $\Delta(A) p:=p\left(F_{A}^{(2 k)}\right)=p\left(F_{A}, F_{A}, \ldots, F_{A}\right)$. We also need $\Delta(A, B) p=2 k \int_{0}^{1} p\left(B-A, F_{A+t(B-A)}^{(2 k-1)}\right) d t$, which is the ChernSimons-Bott form, where $B$ is another $\operatorname{GL}(n, \mathbb{R})$-connection. This introduction of Pontryagin classes, more generally of primary and secondary characteristic classes, is referred to in Chapter 4 of [Vaisman 1987]. It is also helpful to compute them in this paper.

Let $l=n-4 k$ and $\kappa: \bigwedge^{l} H^{1}(M) \rightarrow \mathbb{R}$, the homomorphism defined by

$$
\kappa(a)=\langle p(M) \cup \bigcup a,[M]\rangle / \int_{M} \text { vol for } a \in \wedge^{l} H^{1}(M) .
$$

Lemma 2.2. There exists a homomorphism

$$
\mu: \bigwedge^{l} H^{1}(M) \rightarrow \Omega^{n-1}(M)
$$

such that $d \mu(a)=\kappa(a) \operatorname{vol}-\Delta(A) p \wedge \wedge_{r}$ a for all $a \in \wedge^{l} H^{1}(M)$.

For $\varphi \in \mathscr{D}_{\mathrm{vol}}$ and $a=a_{1} \wedge \cdots \wedge a_{l} \in \wedge^{l} H^{1}(M)$, put

$$
f_{\varphi}(a)=\sum_{m=1}^{l}(-1)^{m-1} \bigwedge_{j=1}^{m-1} \varphi_{\sharp} r\left(a_{j}\right) \wedge f_{\varphi}\left(a_{m}\right) \bigwedge_{j=m+1}^{l} r\left(a_{j}\right)
$$

in $\Omega^{l-1}(M)$ and extend it by linear combination for any $a \in \wedge^{l} H^{1}(M)$. We use the same symbol $f_{\varphi}$ for all $l$, but there is no confusion. Put

$$
J_{\varphi}(a)=\left[\Delta\left(A, \varphi_{*} A\right) p \wedge \bigwedge_{r} a+\Delta\left(\varphi_{*} A\right) p \wedge f_{\varphi}(a)+\varphi_{\sharp} \mu(a)-\mu(a)\right] .
$$

Then it is a well-defined element of $H^{n-1}(M)$. Here, $\varphi_{*} A$ is the pushforward connection of $A$ by $\varphi_{*}: T M \rightarrow T M$. Put

$$
\mathscr{H}=\operatorname{Hom}\left(\bigwedge^{l} H^{1}(M), H^{n-1}(M)\right) .
$$

We can show $J_{\varphi} \in \mathscr{H}$ and the following theorem:

Theorem 2.3. The map

$$
J: \mathscr{D}_{\mathrm{vol}} \ni \varphi \mapsto J_{\varphi} \in \mathscr{H}
$$

is a well-defined crossed homomorphism depending on the choice of $p, A, r$, and $\mu$. Its cohomology class $[J] \in H^{1}\left(\mathscr{D}_{\mathrm{vol}}, \mathscr{H}\right)$ in group cohomology depends only on $p$, not on the choice of $A, r$, and $\mu$. 
Here the action of $\mathscr{D}_{\mathrm{vol}}$ on $\mathscr{H}$ is given by $(\varphi, h) \mapsto \varphi_{\sharp} h$ as mentioned before. By definition, the map $J$ is a crossed homomorphism if and only if the equality $J_{\varphi \psi}=J_{\varphi}+\varphi_{\sharp} J_{\psi}$ holds for all $\varphi, \psi \in \mathscr{D}_{\mathrm{vol}}$. See [Brown 1982] for group cohomology.

In general, the cohomology classes $[J]$ are nontrivial. Proposition 7.8 gives a condition for them to be nontrivial, and Corollary 7.7 is a simple example of them. These are corollaries of Proposition 2.4 below. Since the target $\mathscr{H}$ of $J$ is the same for all $p \in I_{n}^{2 k}$, we obtain the map from $I_{n}^{2 k}$ to $H^{1}\left(\mathscr{D}_{\mathrm{vol}}, \mathscr{H}\right)$ by Theorem 2.3, which is easily checked to be linear, but we don't study this homomorphism in this paper.

In order to state Proposition 2.4, we introduce the volume flux homomorphism. Let $\mathscr{D}_{\mathrm{vol}, 0}$ be the identity component of $\mathscr{D}_{\mathrm{vol}}$, and $\pi: \widetilde{\mathscr{D}}_{\mathrm{vol}, 0} \rightarrow \mathscr{D}_{\mathrm{vol}, 0}$ its universal covering. Each element of $\widetilde{\mathscr{D}}_{\mathrm{vol}, 0}$ is represented by a smooth curve $\left\{\varphi_{s}\right\}_{s \in[0,1]} \subset \mathscr{D}_{\mathrm{vol}, 0}$ with $\varphi_{0}=\mathrm{id}_{M}$, which is denoted by $\left[\varphi_{s}\right] \in \tilde{\mathscr{D}}_{\mathrm{vol}, 0}$. The volume flux homomorphism

$$
\text { Flux }^{\sim}: \widetilde{\mathscr{D}}_{\mathrm{vol}, 0} \rightarrow H^{n-1}(M), \quad \operatorname{Flux}^{\sim}\left(\left[\varphi_{s}\right]\right)=\int_{0}^{1}\left[\iota\left(X_{s}\right) \text { vol }\right] d s
$$

with respect to vol is a well-defined surjective homomorphism [Banyaga 1997]. Here $X_{s}$ is the time-dependent vector field given by $d \varphi_{s} / d s=X_{s} \circ \varphi_{s}$, and $\iota\left(X_{s}\right)$ denotes the interior product by $X_{s}$. The image Flux ${ }^{\sim}\left(\pi^{-1}(\right.$ id) $)$ of the fiber $\pi^{-1}$ (id) $\subset \widetilde{\mathscr{D}}_{\mathrm{vol}, 0}$ at the identity under Flux $\sim$ is called the volume flux group, which is denoted by $\Gamma_{\mathrm{vol}}(M)$.

In order to state the relation of $J$ with Flux ${ }^{\sim}$, we define the homomorphisms

$$
L, L_{+}: H^{n-1}(M) \rightarrow \mathscr{H}
$$

as the linear extensions of

$$
L(w)(a)=p(M) \cup \sum_{m=1}^{l}(-1)^{m-1}\left\langle a_{m} \cup w,[M]\right\rangle \bigcup_{j=1}^{m-1} a_{j} \cup \bigcup_{j=m+1}^{l} a_{j} / \int_{M} \text { vol }
$$

and $L_{+}(w)(a)=-\kappa(a) w+L(w)(a)$ for $w \in H^{n-1}(M)$ and $a=a_{1} \wedge \cdots \wedge a_{l} \in$ $\wedge^{l} H^{1}(M)$.

Proposition 2.4. The equality $J \circ \pi=L_{+} \circ$ Flux $^{\sim}$ holds on $\tilde{\mathscr{D}}_{\mathrm{vol}, 0}$; that is,

$$
J_{\varphi_{1}}(a)=-\kappa(a) \operatorname{Flux}^{\sim}\left(\left[\varphi_{s}\right]\right)+L\left(\operatorname{Flux}^{\sim}\left(\left[\varphi_{s}\right]\right)\right)(a)
$$

holds for any $\left[\varphi_{s}\right] \in \tilde{\mathscr{D}}_{\mathrm{vol}, 0}$ and $a \in \bigwedge^{l} H^{1}(M)$.

Let $L_{p}, L_{p+}$ and $\kappa_{p}$ be the homomorphisms $L, L_{+}$, and $\kappa$ with respect to $p \in$ $I_{n}^{2 k}$ respectively. This proposition implies that the volume flux group $\Gamma_{\mathrm{vol}}(M)$ is contained in the kernel of $L_{p+}$ for all $p$, since $\Gamma_{\text {vol }}(M)$ is defined independently of $p$. In particular, if there exists an $L_{p}$ whose kernel is zero, we have $\Gamma_{\mathrm{vol}}(M)=\{0\}$. The next theorem restates this consequence in terms of $L_{p}$, and its examples are given 
in Corollary 7.6. Let $\mathscr{P}$ be the set of pairs $(p, a)$ of $p \in I_{n}^{2 k}$ and $a \in \bigwedge^{n-4 k} H^{1}(M)$ for some $k$, and $\mathscr{P}_{0}=\left\{(p, a) \in \mathscr{P} \mid \kappa_{p}(a)=0\right\}$. For each $(p, a) \in \mathscr{P}$, we have the homomorphism $L_{p}(\cdot)(a): H^{n-1}(M) \rightarrow H^{n-1}(M)$.

Theorem 2.5. The volume flux group $\Gamma_{\mathrm{vol}}(M)$ of any closed oriented smooth manifold $M$ of dimension $n$ with a volume form vol satisfies

$$
\Gamma_{\mathrm{vol}}(M) \subset\left\{\bigcap_{(p, a) \in \mathscr{P}_{0}} \operatorname{ker} L_{p}(\cdot)(a)\right\} \cap\left\{\bigcap_{(p, a) \in \mathscr{P} \backslash P_{0}} \operatorname{Im} L_{p}(\cdot)(a)\right\} .
$$

Proposition 2.4 tells us that the image of $\mathscr{D}_{\mathrm{vol}, 0}$ by $J$ is contained in $\operatorname{Im} L_{+}$. On the other hand, we know from Moser's result [1965] that the inclusion $\mathscr{D}_{\mathrm{vol}} \hookrightarrow \mathscr{D}$ is a weak homotopy equivalence. Using these, we can show the following theorem:

Theorem 2.6. The crossed homomorphism J descends to a well-defined one;

$$
\mathscr{F}: \pi_{0} \mathscr{D} \rightarrow \mathscr{H} / \operatorname{Im} L_{+} .
$$

Its cohomology class $[\mathscr{E}] \in H^{1}\left(\pi_{0} \mathscr{D}, \mathscr{H} / \operatorname{Im} L_{+}\right)$depends only on $p$, not on the choice of vol, $A, r$ and $\mu$.

As mentioned above, Corollary 7.7 gives a nontrivial example of Theorem 2.3. But since the image of $J$ in this corollary is contained in $\operatorname{Im} L_{+}$, it is a trivial case of Theorem 2.6, so we need a nontrivial example of the cohomology class [\&].

Let $M=\Sigma_{g}$ be a closed oriented surface of genus $g \geqq 3$. The isotopy group $\pi_{0} \mathscr{D}$ is called the mapping class group of $\Sigma_{g}$. We take $p=1 \in I_{2}^{0}$. Then, our crossed homomorphism $J: \mathscr{D}_{\mathrm{vol}} \rightarrow \mathscr{H}=\operatorname{Hom}\left(\wedge^{2} H^{1}\left(\Sigma_{g}\right), H^{1}\left(\Sigma_{g}\right)\right)$ is simply given by $J_{\varphi}(a)=\left[f_{\varphi}(a)+\varphi_{\sharp} \mu(a)-\mu(a)\right]$ for an area form $\omega(=$ vol $)$ on $\Sigma_{g}$, which descends to $\mathscr{F}$ on $\pi_{0} \mathscr{D}$. The subgroup $\mathscr{I}_{g}=\left\{\varphi \in \pi_{0} \mathscr{D} \mid \varphi_{*}=\right.$ id on $\left.H_{1}\left(\Sigma_{g} ; \mathbb{Z}\right)\right\} \subset \pi_{0} \mathscr{D}$ is called the Torelli group. Let $\tau_{g}: \mathscr{I}_{g} \rightarrow \wedge^{3} H_{1}\left(\Sigma_{g} ; \mathbb{Z}\right) / H_{1}\left(\Sigma_{g} ; \mathbb{Z}\right)$ be the first Johnson homomorphism [Johnson 1980]. Using Poincaré duality, we have a natural homomorphism $\bigwedge^{3} H_{1}\left(\Sigma_{g} ; \mathbb{Z}\right) \hookrightarrow \mathscr{H}$. Moreover, we can see that it descends to an injective homomorphism

$$
j: \bigwedge^{3} H_{1}\left(\Sigma_{g} ; \mathbb{Z}\right) / H_{1}\left(\Sigma_{g} ; \mathbb{Z}\right) \hookrightarrow \mathscr{H} / \operatorname{Im} L_{+} .
$$

Theorem 2.7. For any closed oriented surface $\Sigma_{g}$ of genus $g \geqq 3$, the following equality holds:

$$
\left.\mathscr{F}\right|_{\Phi_{g}}=-\frac{1}{2} j \circ \tau_{g} .
$$

We remark that $\left.\mathscr{F}\right|_{\mathscr{I}_{g}}$ is a usual homomorphism because the subgroup $\mathscr{I}_{g}$ of $\pi_{0} \mathscr{D}$ acts trivially on the target of $\mathscr{F}$. This theorem implies that the restriction $\left.\mathscr{F}\right|_{\Phi_{g}}$ is a nontrivial homomorphism, which defines a nontrivial first cohomology class. Thus, the class $[F]$ is also nontrivial. This gives a nontrivial example of Theorem 2.6. 


\section{Lemmas}

In this section we will prepare the lemmas which are needed to show that the homomorphism $J_{\varphi}$ is defined on the exterior product $\wedge^{l} H^{1}(M)$ and that the cohomology class of $J$ is unique.

Let $\alpha_{j} \in Z^{1}(M)$ and $\zeta_{j} \in C^{\infty}(M)$ with $1 \leq j \leq h$. We expand the exterior product $\bigwedge_{j=1}^{h}\left(\alpha_{j}+d \zeta_{j}\right)$, we then have

$$
\bigwedge_{j=1}^{h}\left(\alpha_{j}+d \zeta_{j}\right)-\bigwedge_{j=1}^{h} \alpha_{j}=\sum_{m=1}^{h} \sum_{1 \leq k_{1}<\cdots<k_{m} \leq h} \bigwedge_{p=1}^{m}\left\{\bigwedge_{j=k_{p-1}+1}^{k_{p}-1} \alpha_{j} \wedge d \zeta_{k_{p}}\right\} \wedge \bigwedge_{j=k_{m}+1}^{h} \alpha_{j}
$$

where $k_{0}=0$. Set $\tilde{\alpha}=\alpha_{1} \otimes \cdots \otimes \alpha_{h}, \tilde{\zeta}=\zeta_{1} \otimes \cdots \otimes \zeta_{h}$, and

$$
K_{h}(\tilde{\alpha}, \tilde{\zeta})=\sum_{m=1}^{h} \sum_{1 \leq k_{1}<\cdots<k_{m} \leq h}(-1)^{k_{1}-1} \bigwedge_{j=1}^{k_{1}-1} \alpha_{j} \wedge \zeta_{k_{1}} \bigwedge_{p=2}^{m}\left\{\bigwedge_{j=k_{p-1}+1}^{k_{p}-1} \alpha_{j} \wedge d \zeta_{k_{p}}\right\} \wedge \bigwedge_{j=k_{m}+1}^{h} \alpha_{j}
$$

we then have $K_{h}(\tilde{\alpha}, \tilde{\zeta}) \in \Omega^{h-1}(M)$ and

$$
\bigwedge_{j=1}^{h}\left(\alpha_{j}+d \zeta_{j}\right)-\bigwedge_{j=1}^{h} \alpha_{j}=d K_{h}(\tilde{\alpha}, \tilde{\zeta}) .
$$

This equality is the only requirement for $K_{h}$, so the choice of it is not unique. The following lemma is an easy consequence of the definition of $K_{h}$, so we omit the proof:

Lemma 3.1. The following equalities hold:

(i) $K_{h}(\tilde{\alpha}, \tilde{\zeta}) \wedge \beta=K_{h+1}(\tilde{\alpha} \otimes \beta, \tilde{\zeta} \otimes \eta)-(-1)^{h} \wedge \tilde{\alpha} \cdot \eta-K_{h}(\tilde{\alpha}, \tilde{\zeta}) \wedge d \eta$

(ii) $\beta \wedge K_{h}(\tilde{\alpha}, \tilde{\zeta})=-K_{h+1}(\beta \otimes \tilde{\alpha}, \eta \otimes \tilde{\zeta})+\eta \wedge \tilde{\alpha}+\eta d K_{h}(\tilde{\alpha}, \tilde{\zeta})$.

where $\beta \in Z^{1}(M), \eta \in C^{\infty}(M)$, and $\wedge \tilde{\alpha}=\alpha_{1} \wedge \cdots \wedge \alpha_{h}$.

Let $\mathscr{Y}_{h}$ be the $h$-th symmetric group. Put $\tilde{\alpha}_{\sigma}=\alpha_{\sigma(1)} \otimes \cdots \otimes \alpha_{\sigma(h)}$ and $\tilde{\zeta}_{\sigma}=$ $\zeta_{\sigma(1)} \otimes \cdots \otimes \zeta_{\sigma(h)}$ for $\sigma \in \mathscr{Y}_{h}$.

Lemma 3.2. The equality $K_{h}\left(\tilde{\alpha}_{\sigma}, \tilde{\zeta}_{\sigma}\right) \equiv \operatorname{sgn}(\sigma) K_{h}(\tilde{\alpha}, \tilde{\zeta})\left(\bmod d \Omega^{h-2}(M)\right)$ holds for each $\sigma \in \mathscr{Y}_{h}$.

Proof. It is sufficient to show the equality for any transposition $\sigma=(s, t) \in \mathscr{Y}_{h}$ with $s<t$. For each $1 \leq m \leq h$, put $I_{m}=\left\{\left(k_{1}, \ldots, k_{m}\right) \mid 1 \leq k_{1}<\cdots<k_{m} \leq h\right\}$, and define the map $\sigma: I_{m} \rightarrow I_{m}$ by 


$$
\sigma(u)= \begin{cases}u & \text { if } s, t \notin u, \\ u & \text { if } s, t \in u, \\ \left\{k_{1}, \ldots, k_{m}, t\right\} \backslash\{s\} \text { reordered in ascending order } & \text { if } s \in u, t \notin u, \\ \left\{k_{1}, \ldots, k_{m}, s\right\} \backslash\{t\} \text { reordered in ascending order } & \text { if } s \notin u, t \in u,\end{cases}
$$

where $u=\left(k_{1}, \ldots, k_{m}\right) \in I_{m}$, and $s \in u$ means $s \in\left\{k_{1}, \ldots, k_{m}\right\}$ by abuse of notation. Put

$$
A_{u}=(-1)^{k_{1}-1} \bigwedge_{j=1}^{k_{1}-1} \alpha_{j} \wedge \zeta_{k_{1}} \bigwedge_{p=2}^{m}\left\{\bigwedge_{j=k_{p-1}+1}^{k_{p}-1} \alpha_{j} \wedge d \zeta_{k_{p}}\right\} \wedge \bigwedge_{j=k_{m}+1}^{h} \alpha_{j}
$$

and

$$
A_{u}^{\sigma}=(-1)^{k_{1}-1} \bigwedge_{j=1}^{k_{1}-1} \alpha_{\sigma(j)} \wedge \zeta_{\sigma\left(k_{1}\right)} \bigwedge_{p=2}^{m}\left\{\bigwedge_{j=k_{p-1}+1}^{k_{p}-1} \alpha_{\sigma(j)} \wedge d \zeta_{\sigma\left(k_{p}\right)}\right\} \wedge \bigwedge_{j=k_{m}+1}^{h} \alpha_{\sigma(j)}
$$

for $u=\left(k_{1}, \ldots, k_{m}\right) \in I_{m}$. It is easy to see the following equalities:

(i) If $s, t \notin u$, then $A_{u}^{\sigma}=-A_{\sigma(u)}$.

(ii) If $s, t \in u$, then $A_{u}^{\sigma}=-A_{\sigma(u)}+ \begin{cases}\text { an exact form } & \text { if } s=k_{1}, \\ 0 & \text { if } s \neq k_{1} .\end{cases}$

(iii) If $s \in u, t \notin u$, then $A_{u}^{\sigma}=-A_{\sigma(u)}+ \begin{cases}\text { an exact form } & \text { if } s=k_{1}<k_{2}<t, \\ 0 & \text { otherwise. }\end{cases}$

(iv) If $s \notin u, t \in u$, then $A_{u}^{\sigma}=-A_{\sigma(u)}+ \begin{cases}\text { an exact form } & \text { if } s<k_{1}<k_{2} \leq t, \\ 0 & \text { otherwise. }\end{cases}$

Thus, we have $A_{u}^{\sigma} \equiv-A_{\sigma(u)}\left(\bmod d \Omega^{h-2}(M)\right)$. Since the map $\sigma$ on $I_{m}$ is bijective, we have

$$
\begin{aligned}
K_{h}\left(\tilde{\alpha}_{\sigma}, \tilde{\zeta}_{\sigma}\right) & =\sum_{m=1}^{h} \sum_{u \in I_{m}} A_{u}^{\sigma} \equiv-\sum_{m=1}^{h} \sum_{u \in I_{m}} A_{\sigma(u)}\left(\bmod d \Omega^{h-2}(M)\right) \\
& =-\sum_{m=1}^{h} \sum_{u \in I_{m}} A_{u}=\operatorname{sgn}(\sigma) K_{h}(\tilde{\alpha}, \tilde{\zeta}) .
\end{aligned}
$$

\section{The proofs of Lemmas 2.1 and 2.2}

In this section we will prove Lemmas 2.1 and 2.2 and show some properties of the homomorphisms in these lemmas.

We preserve the notation in Section 2.

Proof of Lemma 2.1. Let $B^{1}(M)$ be the space of smooth exact 1-forms on $M$. We have $\varphi_{\sharp} r(a)-r(a) \in B^{1}(M)$ for any $\varphi \in \mathscr{D}_{\mathrm{vol}}$ and $a \in H^{1}(M)$ since $\left[\varphi_{\sharp} r(a)\right]=a=$ $[r(a)] \in H^{1}(M)$. Since the exterior derivative $d$ gives an isomorphism from $C_{0}^{\infty}(M)$ 
to $B^{1}(M)$, we have a unique $f_{\varphi}(a) \in C_{0}^{\infty}(M)$ satisfying $d f_{\varphi}(a)=\varphi_{\sharp} r(a)-r(a)$. The equality

$$
(\varphi \psi)_{\sharp} r(a)-r(a)=\varphi_{\sharp} r(a)-r(a)+\varphi_{*}\left(\psi_{\sharp} r-r\right)\left(\varphi^{*} a\right)
$$

for $\varphi, \psi \in \mathscr{D}_{\mathrm{vol}}$ and $a \in H^{1}(M)$, and the injectivity of $d$ on $C_{0}^{\infty}(M)$ imply $f_{\varphi \psi}(a)=$ $f_{\varphi}(a)+\left(\varphi_{\sharp} f_{\psi}\right)(a)$.

Let $u: H^{1}(M) \rightarrow Z^{1}(M)$ be another linear section of the projection $Z^{1}(M) \rightarrow$ $H^{1}(M)$. We have two crossed homomorphisms $f_{r}$ and $f_{u}$ in Lemma 2.1 with respect to $r$ and $u$ respectively. The proof of the following lemma is almost the same as that of Lemma 2.1, so we omit it:

Lemma 4.1. There is a unique homomorphism

$$
q=q_{r u}: H^{1}(M) \rightarrow C_{0}^{\infty}(M)
$$

such that $d q(a)=u(a)-r(a)$ for all $a \in H^{1}(M)$. Moreover, the equality

$$
f_{u, \varphi}-f_{r, \varphi}=\varphi_{\sharp} q-q
$$

holds in $\operatorname{Hom}\left(H^{1}(M), C_{0}^{\infty}(M)\right)$.

Proof of Lemma 2.2. Note that $\kappa(a)$ vol $-\Delta(A) p \wedge \wedge_{r} a \in \Omega^{n}(M)$ is exact for any $a \in \Lambda^{l} H^{1}(M)$ by the definition of $\kappa$. Fix a basis $\left\{e_{i}\right\} \subset \wedge^{l} H^{1}(M)$ and take $\mu\left(e_{i}\right) \in \Omega^{n-1}(M)$ arbitrarily such that $d \mu\left(e_{i}\right)=\kappa\left(e_{i}\right)$ vol $-\Delta(A) \wedge \wedge_{r} e_{i}$ for all $i$. The linear combination of $\mu\left(e_{i}\right)$ 's give the required homomorphism.

For each $\tilde{a}=a_{1} \otimes \cdots \otimes a_{h} \in \otimes^{h} H^{1}(M)$, set

$$
\mathfrak{K}_{h}(\tilde{a})=K_{h}\left(r\left(a_{1}\right) \otimes \cdots \otimes r\left(a_{h}\right), q\left(a_{1}\right) \otimes \cdots \otimes q\left(a_{h}\right)\right),
$$

where $r$ and $q$ are the homomorphisms in Lemma 2.1 and 4.1 respectively. Then the linear extension defines a homomorphism

$$
\mathfrak{K}_{h}: \bigotimes^{h} H^{1}(M) \rightarrow \Omega^{h-1}(M)
$$

for each $h$. The composition $\otimes^{h} H^{1}(M) \rightarrow \bigwedge^{h} H^{1}(M) \stackrel{\bigwedge_{r}}{\longrightarrow} \Omega^{h}(M)$ is also denoted by the same symbol, $\bigwedge_{r}$, where the first homomorphism is the projection $\otimes^{h} H^{1}(M) \rightarrow \wedge^{h} H^{1}(M)$ given by $\tilde{a}=a_{1} \otimes \cdots \otimes a_{h} \mapsto a=a_{1} \wedge \cdots \wedge a_{h}$.

Similarly, we use the same symbol for a homomorphism on $\wedge^{h} H^{1}(M)$ and the composition of it with the projection $\bigotimes^{h} H^{1}(M) \rightarrow \bigwedge^{h} H^{1}(M)$. For example, the image of $\tilde{a}$ by the composition $\bigotimes^{l} H^{1}(M) \rightarrow \wedge^{l} H^{1}(M) \stackrel{\mu}{\rightarrow} \Omega^{n-1}(M)$ is denoted by $\mu(\tilde{a}):=\mu(a)$. There is no confusion since we can distinguish them by $a$ or $\tilde{a}$.

For each permutation $\sigma \in \mathscr{S}_{h}$, let $\bigotimes^{h} H^{1}(M) \ni \tilde{a} \mapsto \tilde{a}_{\sigma} \in \bigotimes^{h} H^{1}(M)$ be the linear extension of $a_{1} \otimes \cdots \otimes a_{h} \mapsto a_{\sigma(1)} \otimes \cdots \otimes a_{\sigma(h)}$. 
Lemma 4.2. Let $\tilde{a} \in \bigotimes^{h} H^{1}(M), b \in H^{1}(M)$, and $\varphi \in \mathscr{D}_{\mathrm{vol}}$. Then:

(i) $\bigwedge_{u} \tilde{a}-\bigwedge_{r} \tilde{a}=d \mathfrak{K}_{h}(\tilde{a})$.

(ii) $\varphi_{\sharp} \mathfrak{K}_{r u, h}=\mathfrak{K}_{\varphi_{\sharp} r \varphi_{\sharp} u, h}$, where $\mathfrak{K}_{r u, h}$ and $\mathfrak{K}_{\varphi_{\sharp} r \varphi_{\sharp} u, h}$ are $\mathfrak{K}_{h}$ with respect to $r$, $u$, and $\varphi_{\sharp} r, \varphi_{\sharp} u$ respectively.

(iii) $r(b) \wedge \mathfrak{K}_{h}(\tilde{a})=-\mathfrak{K}_{h+1}(b \otimes \tilde{a})+q(b) \wedge_{r} \tilde{a}+q(b) d \mathfrak{K}_{h}(\tilde{a})$.

(iv) $\mathfrak{K}_{h}(\tilde{a}) \wedge r(b)=\mathfrak{K}_{h+1}(\tilde{a} \otimes b)-(-1)^{h} \bigwedge_{r} \tilde{a} \cdot q(b)-\mathfrak{K}_{h}(\tilde{a}) \wedge d q(b)$.

(v) $\varphi_{\sharp} \mathfrak{K}_{h}(\tilde{a}) \wedge \varphi_{\sharp} r(b)=\varphi_{\sharp} \mathfrak{K}_{h+1}(\tilde{a} \otimes b)-(-1)^{h} \bigwedge_{\varphi_{\sharp} r} \tilde{a} \cdot \varphi_{\sharp} q(b)-\varphi_{\sharp} \mathfrak{K}_{h}(\tilde{a}) \wedge d \varphi_{\sharp} q(b)$.

(vi) $\mathfrak{K}_{h}\left(\tilde{a}_{\sigma}\right) \equiv \operatorname{sgn}(\sigma) \mathfrak{K}_{h}(\tilde{a})\left(\bmod d \Omega^{h-2}(M)\right)$ for each $\sigma \in \mathscr{Y}_{h}$.

Proof. (i) and (ii) follow immediately from the definition of $\mathfrak{K}_{h}$. (iii), (iv), (v), and (vi) are direct consequences of Lemmas 3.1 and 3.2.

\section{Bott homomorphisms}

In this section we shall recall Bott homomorphisms [Vaisman 1987], which are useful for our computation.

Let $G$ be a Lie group, but we consider only $G=\mathrm{GL}(n, \mathbb{R})$ in this paper. Let $\pi: P \rightarrow M$ be a principal $G$-bundle over a manifold $M$. Let $A_{h}$ with $h=0,1, \ldots, r$ be $r+1$ connection forms on $P$, and

$$
\Delta^{r}:=\left\{\left(t_{0}, t_{1}, \ldots, t_{r}\right) \in \mathbb{R}^{r+1} \mid t_{h} \geqq 0, \sum_{h=0}^{r} t_{h}=1\right\}
$$

the standard $r$-simplex. Then we have the average connection $\tilde{A}=\sum_{h=0}^{r} t_{h} A_{h}$ on the product bundle $\pi \times$ id: $P \times \Delta^{r} \rightarrow M \times \Delta^{r}$. Let $I^{k}(G)\left(=I_{n}^{k}\right)$ be the vector space of invariant, symmetric, multilinear functions on the $k$-th product $\mathfrak{g}^{k}$ of the Lie algebra $\mathfrak{g}$ of $G$ with values in $\mathbb{R}$. For each $p \in I^{k}(G)$, put $\Delta\left(A_{0}, \ldots, A_{r}\right) p=$ $(-1)^{\frac{r+1}{2}} \int_{\Delta^{r}} p\left(F_{\tilde{A}}^{(k)}\right)$, where $F_{\tilde{A}}$ is the curvature form of $\tilde{A}$, the orientation of $\Delta^{r}$ is given by $d t_{1} \wedge \cdots \wedge d t_{r}$ with $t_{0}=1-\sum_{h=1}^{r} t_{h}$, and $p\left(F_{\tilde{A}}, F_{\tilde{A}}, \ldots, F_{\tilde{A}}\right)$ is denoted by $p\left(F_{\tilde{A}}^{(k)}\right)$. Then we have Bott homomorphisms $\Delta\left(A_{0}, \ldots, A_{r}\right): I^{k}(G) \rightarrow \Omega^{2 k-r}(M)$. They have the following properties:

(i) $d \Delta\left(A_{0}, \ldots, A_{r}\right) p=\sum_{h=0}^{r}(-1)^{h} \Delta\left(A_{0}, \ldots, A_{h-1}, A_{h+1}, \ldots, A_{r}\right) p$, in particular, $d \Delta\left(A_{0}\right) p=0$ for $r=0$.

(ii) $\Delta\left(A_{0}\right): I^{k}(G) \rightarrow \Omega^{*}(M)$ is the Chern-Weil homomorphism; that is, the equality $\Delta\left(A_{0}\right) p=p\left(F_{A_{0}}^{(k)}\right)$ holds.

(iii) $\Delta\left(A_{0}, A_{1}\right) p=k \int_{0}^{1} p\left(\alpha, F_{A_{t}}^{(k-1)}\right) d t$, where $\alpha=A_{1}-A_{0}$ and $A_{t}=A_{0}+t \alpha$. Let $Q$ be another principal $G$-bundle over $M$, and $\tilde{\varphi}: Q \rightarrow P$ a $G$-bundle isomorphism over a diffeomorphism $\varphi$ of $M$. The pushforward connection of $A$ by $\tilde{\varphi}$ is denoted by $\tilde{\varphi}_{*} A:=\left(\tilde{\varphi}^{-1}\right)^{*} A$.

(iv) $\Delta\left(\tilde{\varphi}_{*} A_{0}, \tilde{\varphi}_{*} A_{1}, \ldots, \tilde{\varphi}_{*} A_{r}\right) p=\varphi_{*} \Delta\left(A_{0}, A_{1}, \ldots, A_{r}\right) p$. 


\section{Proof of Theorem 2.3}

Recall that for any integer $h$ and any homomorphism $v: H^{1}(M) \rightarrow Z^{1}(M)$, $\bigwedge_{v}: \wedge^{h} H^{1}(M) \rightarrow \Omega^{h}(M)$ is defined as the linear extension of $a_{1} \wedge \cdots \wedge a_{h} \mapsto$ $v\left(a_{1}\right) \wedge \cdots \wedge v\left(a_{h}\right)$. We use the same symbol $\wedge_{v}$ for the composition $\otimes^{h} H^{1}(M) \rightarrow$ $\wedge^{h} H^{1}(M) \rightarrow \Omega^{h}(M)$ as mentioned above. We consider mainly the cases of $v=r, u, \varphi_{\sharp} r, \ldots$

For any $\varphi, \psi \in \mathscr{D}_{\mathrm{vol}}$ and $\tilde{a}=a_{1} \otimes \cdots \otimes a_{l} \in \otimes^{l} H^{1}(M)$, put

$$
f_{\varphi}(\tilde{a})=\sum_{m=1}^{l}(-1)^{m-1} \bigwedge_{\varphi_{\sharp} r} \tilde{a}_{1, m-1} \wedge f_{\varphi}\left(a_{m}\right) \bigwedge_{r} \tilde{a}_{m+1, l}
$$

and

$f_{\varphi, \psi}(\tilde{a})$

$$
=-\sum_{1 \leq k<m \leq l}(-1)^{m+k} \bigwedge_{(\varphi \psi)_{\sharp}} \tilde{a}_{1, k-1} \wedge \varphi_{\sharp}\left(f_{\psi}\right)\left(a_{k}\right) \bigwedge_{\varphi_{\sharp} r} \tilde{a}_{k+1, m-1} \wedge f_{\varphi}\left(a_{m}\right) \wedge_{r} \tilde{a}_{m+1, l},
$$

where $\tilde{a}_{i, j}=a_{i} \otimes \cdots \otimes a_{j}$ with $i<j$ and $\bigwedge_{*} a_{i+1, i}=1$. Linear extension defines the maps

$$
\mathscr{D}_{\mathrm{vol}} \ni \varphi \mapsto f_{\varphi} \in \operatorname{Hom}\left(\otimes^{l} H^{1}(M), \Omega^{l-1}(M)\right)
$$

and

$$
\mathscr{D}_{\mathrm{vol}}^{2} \ni(\varphi, \psi) \mapsto f_{\varphi, \psi} \in \operatorname{Hom}\left(\otimes^{l} H^{1}(M), \Omega^{l-2}(M)\right) .
$$

Lemma 6.1. For any $\varphi, \psi \in \mathscr{D}_{\mathrm{vol}}$, and $\tilde{a} \in \bigotimes^{l} H^{1}(M)$, the following equalities hold:

(i) $d f_{\varphi}(\tilde{a})=\bigwedge_{\varphi_{\sharp} r} \tilde{a}-\bigwedge_{r} \tilde{a}$.

(ii) $d \varphi_{\sharp}\left(f_{\psi}\right)(\tilde{a})=\bigwedge_{(\varphi \psi)_{\sharp} r} \tilde{a}-\bigwedge_{\varphi_{\sharp} r} \tilde{a}$.

(iii) $\varphi_{\sharp} f_{\psi}(\tilde{a})-f_{\varphi \psi}(\tilde{a})+f_{\varphi}(\tilde{a})=d f_{\varphi, \psi}(\tilde{a})$.

Proof. For (i), it is sufficient to show the equality for $\tilde{a}=a_{1} \otimes \cdots \otimes a_{l} \in \bigotimes^{l} H^{1}(M)$. Direct computation shows

$$
\begin{aligned}
d f_{\varphi}(\tilde{a}) & =\sum_{m=1}^{l} \bigwedge_{\varphi_{\sharp}} \tilde{a}_{1, m-1} \wedge d f_{\varphi}\left(a_{m}\right) \wedge \bigwedge_{r} \tilde{a}_{m+1, l} \\
& =\sum_{m=1}^{l} \bigwedge_{\varphi_{\sharp} r} \tilde{a}_{1, m-1} \wedge\left\{\varphi_{\sharp} r\left(a_{m}\right)-r\left(a_{m}\right)\right\} \wedge \bigwedge_{r} \tilde{a}_{m+1, l} \\
& =\sum_{m=1}^{l} \bigwedge_{\varphi_{\sharp} r} \tilde{a}_{1, m} \wedge \bigwedge_{r} \tilde{a}_{m+1, l}-\sum_{m=1}^{l} \bigwedge_{\varphi_{\sharp} r} \tilde{a}_{1, m-1} \wedge \bigwedge_{r} \tilde{a}_{m, l} \\
& =\bigwedge_{\varphi_{\sharp} r} \tilde{a}-\bigwedge_{r} \tilde{a} .
\end{aligned}
$$


A similar computation to (i) shows (ii) and (iii), so we omit it.

Lemma 6.2. For any $\varphi \in \mathscr{D}_{\mathrm{vol}}$ and $\tilde{a}=a_{1} \otimes \cdots \otimes a_{l} \in \otimes^{l} H^{1}(M)$, the following equalities hold:

(i) $f_{\varphi}(\tilde{a})=f_{\varphi}\left(\tilde{a}_{1, l-1}\right) \wedge r\left(a_{l}\right)+(-1)^{l-1} \wedge_{\varphi_{\sharp} r} \tilde{a}_{1, l-1} \wedge f_{\varphi}\left(a_{l}\right)$.

(ii) $f_{\varphi}(\tilde{a})=f_{\varphi}\left(a_{1}\right) \wedge_{r} \tilde{a}_{2, l}-\varphi_{\sharp} r\left(a_{1}\right) \wedge f_{\varphi}\left(\tilde{a}_{2, l}\right)$.

(iii) $f_{\varphi}\left(\tilde{a}_{\sigma}\right)=\operatorname{sgn}(\sigma) f_{\varphi}(\tilde{a})+$ an exact form, where $\sigma \in \mathscr{S}_{l}$ is any permutation of degree $l$ and $\tilde{a}_{\sigma}=a_{\sigma(1)} \otimes \cdots \otimes a_{\sigma(l)}$.

Proof. (i) and (ii) directly follow from the definition of $f$.

For (iii), we proceed by induction on $l$. It is trivial for $l=1$. For $l=2$ and $\sigma=(1,2) \in \mathscr{Y}_{2}$, the equality $f_{\varphi}\left(a_{1} \otimes a_{2}\right)+f_{\varphi}\left(a_{2} \otimes a_{1}\right)=d\left\{-f_{\varphi}\left(a_{1}\right) f_{\varphi}\left(a_{2}\right)\right\}$ is a desired one. Assume that the statement holds for $l-1(\geqq 1)$. Let $\sigma \in \mathscr{S}_{l}$. To begin with, we consider the case of $\sigma(l)=l$. We can consider $\sigma$ as an element of $\mathscr{Y}_{l-1}$. By assumption, there exists $h \in \Omega^{l-3}(M)$ such that $f_{\varphi}\left(\tilde{a}_{\sigma\{1, l-1\}}\right)=$ $\operatorname{sgn}(\sigma) f_{\varphi}\left(\tilde{a}_{1, l-1}\right)+d h$, where $\tilde{a}_{\sigma\{1, l-1\}}=a_{\sigma(1)} \otimes \cdots \otimes a_{\sigma(l-1)}$. Using (i), we have

$$
\begin{aligned}
f_{\varphi}\left(\tilde{a}_{\sigma}\right) & =f_{\varphi}\left(\tilde{a}_{\sigma\{1, l-1\}} \otimes a_{l}\right) \\
& =f_{\varphi}\left(\tilde{a}_{\sigma\{1, l-1\}}\right) \wedge r\left(a_{l}\right)+(-1)^{l-1} \bigwedge_{\varphi_{\sharp} r} \tilde{a}_{\sigma\{1, l-1\}} \wedge f_{\varphi}\left(a_{l}\right) \\
& =\left\{\operatorname{sgn}(\sigma) f_{\varphi}\left(\tilde{a}_{1, l-1}\right)+d h\right\} \wedge r\left(a_{l}\right)+(-1)^{l-1} \operatorname{sgn}(\sigma) \wedge_{\varphi_{\sharp}} \tilde{a}_{1, l-1} \wedge f_{\varphi}\left(a_{l}\right) \\
& =\operatorname{sgn}(\sigma) f_{\varphi}(\tilde{a})+d\left\{h \wedge r\left(a_{l}\right)\right\} .
\end{aligned}
$$

In the other cases we consider that $\sigma$ can be given as the product of a transposition and a permutation of essentially lower degree such as the case above. Using this and induction hypothesis we can show the $l$ case. By induction, we complete the proof of (iii).

For each $\varphi \in \mathscr{D}_{\mathrm{vol}}$, we define the map

$$
\tilde{J}_{\varphi}: \bigotimes^{l} H^{1}(M) \rightarrow \Omega^{n-1}(M)
$$

by

$$
\tilde{J}_{\varphi}(\tilde{a})=\Delta\left(A, \varphi_{*} A\right) p \wedge \bigwedge_{r} \tilde{a}+\Delta\left(\varphi_{*} A\right) p \wedge f_{\varphi}(\tilde{a})+\varphi_{\sharp} \mu(\tilde{a})-\mu(\tilde{a})
$$

for each $\tilde{a} \in \bigotimes^{l} H^{1}(M)$.

Lemma 6.3. (i) $d \tilde{J}_{\varphi}(\tilde{a})=0$ for any $\varphi \in \mathscr{D}_{\mathrm{vol}}$ and $\tilde{a} \in \bigotimes^{l} H^{1}(M)$. Put $J_{\varphi}(\tilde{a})=$ $\left[\tilde{J}_{\varphi}(\tilde{a})\right]$.

(ii) The map $J_{\varphi}: \bigotimes^{l} H^{1}(M) \rightarrow H^{n-1}(M)$ is a homomorphism.

(iii) The map $J: \mathscr{D}_{\mathrm{vol}} \ni \varphi \mapsto J_{\varphi} \in \operatorname{Hom}\left(\bigotimes^{l} H^{1}(M), H^{n-1}(M)\right)$ is a crossed homomorphism. 
Proof. (i) Using the definitions, Lemma 2.2, Lemma 6.1, and properties of Bott homomorphisms, we have

$$
\begin{aligned}
d \tilde{J}_{\varphi}(\tilde{a})= & \left\{\Delta\left(\varphi_{*} A\right) p-\Delta(A) p\right\} \wedge \wedge_{r} \tilde{a}+\Delta\left(\varphi_{*} A\right) p \wedge\left\{\bigwedge_{\varphi_{\sharp} r} \tilde{a}-\bigwedge_{r} \tilde{a}\right\} \\
& \quad+\varphi_{*}\left\{\kappa\left(\varphi^{*} \tilde{a}\right) \operatorname{vol}-\Delta(A) p \wedge \bigwedge_{r} \varphi^{*} \tilde{a}\right\}-\left\{\kappa(\tilde{a}) \operatorname{vol}-\Delta(A) p \wedge \wedge_{r} \tilde{a}\right\} \\
= & 0 .
\end{aligned}
$$

(ii) By (i), $\tilde{a} \mapsto J_{\varphi}(\tilde{a})$ is well-defined as a map. Its linearity is obvious.

(iii) Direct computation using Lemma 6.1 and properties of Bott homomorphisms shows the equality

$$
\begin{aligned}
\varphi_{\sharp} \tilde{J}_{\psi}(\tilde{a})-\tilde{J}_{\varphi \psi}(\tilde{a})+ & \tilde{J}_{\varphi}(\tilde{a}) \\
= & d\left[\Delta\left(A, \varphi_{*} A,(\varphi \psi)_{*} A\right) p \wedge \Lambda_{r} \tilde{a}+\Delta\left((\varphi \psi)_{*} A\right) p \wedge f_{\varphi, \psi}(\tilde{a})\right. \\
& \left.-\Delta\left(\varphi_{*} A,(\varphi \psi)_{*} A\right) p \wedge f_{\varphi}(\tilde{a})\right]
\end{aligned}
$$

in $\Omega^{n-1}(M)$. This implies that the map $J$ is a crossed homomorphism.

Let $B$ be another $\operatorname{GL}(n, \mathbb{R})$-connection on $T M$ and $u: H^{1}(M) \rightarrow Z^{1}(M)$ another linear section of the projection $Z^{1}(M) \rightarrow H^{1}(M)$. Let

$$
\tilde{v}: \bigotimes^{l} H^{1}(M) \rightarrow \Omega^{n-1}(M)
$$

be the homomorphism defined by

$$
\tilde{v}(\tilde{a})=\mu_{T}(\tilde{a})-\mu_{S}(\tilde{a})+\Delta(A, B) p \wedge \wedge_{u} \tilde{a}+\Delta(A) p \wedge \mathfrak{K}_{l}(\tilde{a})
$$

for each $\tilde{a} \in \bigotimes^{l} H^{1}(M)$. Here $\mu_{T}$ and $\mu_{S}$ are $\mu$ 's in Lemma 2.2 with respect to $T=\{p, B, u\}$ and $S=\{p, A, r\}$ respectively.

Lemma 6.4. (i) $d \tilde{v}(\tilde{a})=0$ for any $\tilde{a} \in \bigotimes^{l} H^{1}(M)$.

(ii) The map

$$
v: \bigotimes^{l} H^{1}(M) \ni \tilde{a} \mapsto[\tilde{v}(\tilde{a})] \in H^{n-1}(M)
$$

is a well-defined homomorphism.

Proof. (i) Using Lemma 2.2, Lemma 4.2, and properties of the Bott homomorphisms, we have

$$
\begin{aligned}
d \tilde{v}(\tilde{a})= & \kappa(\tilde{a}) \operatorname{vol}-\Delta(B) p \wedge \bigwedge_{u} \tilde{a}-\left\{\kappa(\tilde{a}) \operatorname{vol}-\Delta(A) p \wedge \wedge_{r} \tilde{a}\right\} \\
& +\{\Delta(B) p-\Delta(A) p\} \wedge \wedge_{u} \tilde{a}+\Delta(A) p \wedge\left\{\bigwedge_{u} \tilde{a}-\bigwedge_{r} \tilde{a}\right\} \\
& =0 .
\end{aligned}
$$

(ii) By (i), $v$ is defined as a map and its linearity is clear. 
$\tilde{J}_{\varphi}(\tilde{a})$ is written as

$$
\tilde{J}_{S, \varphi}(\tilde{a})=\Delta\left(A, \varphi_{*} A\right) p \wedge \bigwedge_{r} \tilde{a}+\Delta\left(\varphi_{*} A\right) p \wedge f_{r, \varphi}(\tilde{a})+\varphi_{\sharp} \mu_{S}(\tilde{a})-\mu_{S}(\tilde{a})
$$

for the choice $S=\left\{p, A, r, \mu_{S}\right\}$ of the ingredients to define it. $S$ is doubly used, but there is no confusion, and $f_{r}$ denotes the $f$ in Lemma 2.1 with respect to $r$. We consider also

$$
\tilde{J}_{T, \varphi}(\tilde{a})=\Delta\left(B, \varphi_{*} B\right) p \wedge \bigwedge_{u} \tilde{a}+\Delta\left(\varphi_{*} B\right) p \wedge f_{u, \varphi}(\tilde{a})+\varphi_{\sharp} \mu_{T}(\tilde{a})-\mu_{T}(\tilde{a})
$$

with respect to another choice $T=\left\{p, B, u, \mu_{T}\right\}$. We note that $p$ in $S$ and $T$ is common.

Lemma 6.5. For any $\varphi \in \mathscr{D}_{\mathrm{vol}}$ and $\tilde{a} \in \bigotimes^{l} H^{1}(M)$, the following equality holds:

$$
\tilde{J}_{T, \varphi}(\tilde{a})-\tilde{J}_{S, \varphi}(\tilde{a})=\varphi_{\sharp} \tilde{v}(\tilde{a})-\tilde{v}(\tilde{a})+\text { an exact form. }
$$

Proof. It is sufficient to show the following equality for $\tilde{a}=a_{1} \otimes \cdots \otimes a_{l}$ with $a_{i} \in H^{1}(M)$ :

$$
\begin{aligned}
& \tilde{J}_{T, \varphi}(\tilde{a})- \tilde{J}_{S, \varphi}(\tilde{a})-\left\{\varphi_{\sharp} \tilde{v}(\tilde{a})-\tilde{v}(\tilde{a})\right\} \\
&=d\left(\left\{\Delta\left(A, B, \varphi_{*} B\right) p-\Delta\left(A, \varphi_{*} A, \varphi_{*} B\right) p\right\} \wedge \wedge_{u} \tilde{a}\right. \\
&+(-1)^{4 k-1} \Delta\left(A, \varphi_{*} A\right) p \wedge \mathfrak{K}_{l}(\tilde{a})-(-1)^{4 k-1}\left\{\varphi_{*} \Delta(A, B) p\right\} \wedge f_{u, \varphi}(\tilde{a}) \\
&+\Delta\left(\varphi_{*} A\right) p \wedge {\left[\sum _ { m = 1 } ^ { l } ( - 1 ) ^ { m - 1 } \left\{\varphi_{\sharp} \mathfrak{K}_{m-1}\left(\tilde{a}_{1, m-1}\right) \wedge f_{u, \varphi}\left(a_{m}\right) \wedge_{u} \tilde{a}_{m+1, l}\right.\right.} \\
&\left.\left.\left.+(-1)^{m-1} \bigwedge_{\varphi_{\sharp}} \tilde{a}_{1, m-1} \wedge f_{r, \varphi}\left(a_{m}\right) \mathfrak{K}_{l-m}\left(\tilde{a}_{m+1, l}\right)\right\}\right]\right) .
\end{aligned}
$$

Here we note $\mathfrak{K}_{0}(*)=0$ and $\bigwedge_{* *} \tilde{a}_{*+1, *}=1$.

It is easy to show that the difference of both sides of the expression is equal to 0 . The computation is carried out by using Lemmas 2.1, 4.1, 4.2, and 6.1. But it is standard, so we omit it.

Proof of Theorem 2.3. For any $\varphi \in \mathscr{D}_{\mathrm{vol}}$, the homomorphism $J_{\varphi}$ defined in Lemma 6.3 descends to the homomorphism $J_{\varphi}: \wedge^{l} H^{1}(M) \rightarrow H^{n-1}(M)$ by Lemma 6.2(iii) and the closedness of $\Delta(A) p$. Here we use the same symbol $J_{\varphi}$ for different domains $\bigotimes^{l} H^{1}(M)$ and $\wedge^{l} H^{1}(M)$ as mentioned before. Lemma 6.3 implies that the map $J: \mathscr{D}_{\mathrm{vol}} \rightarrow \mathscr{H}$ is a crossed homomorphism. Since a crossed homomorphism is a 1-cocycle in group cohomology theory, we have the cohomology class $[J] \in$ $H^{1}\left(\mathscr{D}_{\mathrm{vol}}, \mathscr{H}\right)$ in group cohomology [Brown 1982]. Similarly, the homomorphism $v$ defined in Lemma 6.4(ii) descends to a homomorphism $v \in \mathscr{H}$ by Lemma 4.2(vi). For any $S=\left\{p, A, r, \mu_{S}\right\}$ and $T=\left\{p, B, u, \mu_{T}\right\}$ with common $p$, Lemma 6.5 
implies $J_{T, \varphi}-J_{S, \varphi}=\varphi_{\sharp} v-v$; hence, $J_{T}-J_{S}=\delta v$ as 1-cocycles in $C^{1}\left(\mathscr{D}_{\mathrm{vol}}, \mathscr{H}\right)$, where $\mathscr{H}=C^{0}\left(\mathscr{D}_{\mathrm{vol}}, \mathscr{H}\right) \stackrel{\delta}{\rightarrow} C^{1}\left(\mathscr{D}_{\mathrm{vol}}, \mathscr{H}\right) \stackrel{\delta}{\rightarrow} \cdots$ is the cochain complex. Thus, we have the independence of $[J]$ from the choice of $S$ except for $p \in S$.

\section{The derivative of $J$}

In this section we will compute the derivative of $J$ along curves in $\mathscr{D}_{\mathrm{vol}}$ and show some results related to volume flux homomorphisms and groups.

Let $\left\{\varphi_{s}\right\}_{s \in[0,1]} \subset \mathscr{D}_{\mathrm{vol}}$ be any smooth curve and $X_{s}$ the time-dependent vector field on $M$ defined by $d \varphi_{s} / d s=X_{s} \circ \varphi_{s}$. Let

$$
I: C^{\infty}(M) \rightarrow \mathbb{R}
$$

be the homomorphism defined by $I(h)=\int_{M} h$ vol $/ \int_{M}$ vol for $h \in C^{\infty}(M)$. Recall that $f$ is the crossed homomorphism defined in Lemma 2.1.

Lemma 7.1. $d f_{\varphi_{s}}(a) / d s=-\iota\left(X_{s}\right) \varphi_{s \sharp} r(a)+I\left(\iota\left(X_{s}\right) \varphi_{s \sharp} r(a)\right)$ for any $a \in H^{1}(M)$.

Proof. Put $Y_{s}:=-\left(\varphi_{s}^{-1}\right)_{*} X_{s}$, where $(\cdot)_{*}$ denotes the push-forward of vector fields. It satisfies $d \varphi_{s}^{-1} / d s=Y_{s} \circ \varphi_{s}^{-1}$. Set $b:=\varphi_{s}^{*} a \in H^{1}(M)$. It is constant with respect to $s$ and we have $d f_{\varphi_{s}}(a)=\left(\varphi_{s}^{-1}\right)^{*} r(b)-r(a)$. Using Cartan's formula for the Lie derivative $\mathscr{L}$ of differential forms, we have

$$
\begin{aligned}
d\left\{\frac{d}{d s} f_{\varphi_{s}}(a)\right\} & =\frac{d}{d s}\left\{\left(\varphi_{s}^{-1}\right)^{*} r(b)\right\}=\left(\varphi_{s}^{-1}\right)^{*} \mathscr{L}_{Y_{s}} r(b) \\
& =\left(\varphi_{s}^{-1}\right)^{*}\left\{d \iota\left(Y_{s}\right) r(b)+\iota\left(Y_{S}\right) d r(b)\right\}=-d\left\{\iota\left(X_{s}\right) \varphi_{s *} r(b)\right\} \\
& =d\left\{-\iota\left(X_{s}\right) \varphi_{s \sharp} r(a)+I\left(\iota\left(X_{s}\right) \varphi_{s \sharp} r(a)\right)\right\} .
\end{aligned}
$$

Since $\left(d f_{\varphi_{s}} / d s\right)(a)$ and $-\iota\left(X_{s}\right) \varphi_{s \sharp} r(a)+I\left(\iota\left(X_{s}\right) \varphi_{s \sharp} r(a)\right)$ belong to $C_{0}^{\infty}(M)$, we obtain the lemma.

In the following lemma, $f_{\varphi}$ and $\mu$ are the homomorphisms from $\otimes^{l} H^{1}(M)$ to $\Omega^{n-1}(M)$ :

Lemma 7.2. For any $\tilde{a}_{1, l}=a_{1} \otimes \cdots \otimes a_{l}$ and $\tilde{a} \in \otimes^{l} H^{1}(M)$, the following equalities hold:

(i) $\frac{d}{d s} f_{\varphi_{s}}\left(\tilde{a}_{1, l}\right)=d\left\{\sum_{m=1}^{l}(-1)^{m} \iota\left(X_{s}\right)\left(\bigwedge_{\varphi_{s \sharp r}} \tilde{a}_{1, m-1}\right) \wedge f_{\varphi_{s}}\left(a_{m}\right) \wedge_{r} \tilde{a}_{m+1, l}\right\}$

$$
\begin{array}{r}
+\sum_{m=1}^{l}(-1)^{m-1} \bigwedge_{\varphi_{s \sharp r} r} \tilde{a}_{1, m-1} \wedge I\left(\iota\left(X_{s}\right) \varphi_{s \sharp} r\left(a_{m}\right)\right) \bigwedge_{r} \tilde{a}_{m+1, l} \\
-\iota\left(X_{s}\right)\left(\bigwedge_{\varphi_{s \sharp}} \tilde{a}_{1, l}\right) .
\end{array}
$$

(ii) $\frac{d}{d s}\left\{\varphi_{s \sharp} \mu(\tilde{a})-\mu(\tilde{a})\right\}=-\kappa(\tilde{a}) \iota\left(X_{s}\right) \operatorname{vol}+\left\{\iota\left(X_{s}\right) \Delta\left(\varphi_{s *} A\right) p\right\} \wedge \bigwedge_{\varphi_{s \sharp}} \tilde{a}$

$$
+\Delta\left(\varphi_{s *} A\right) p \wedge \iota\left(X_{s}\right)\left(\bigwedge_{\varphi_{s \sharp r}} \tilde{a}\right)-d\left\{\iota\left(X_{s}\right) \varphi_{s \sharp} \mu(\tilde{a})\right\} .
$$


Proof. We note that $\varphi_{s}^{*} a_{j} \in H^{1}(M)$ is constant with respect to $s$.

(i) Similar to the proof of Lemma 7.1, we get

$$
\frac{d}{d s}\left(\bigwedge_{\varphi_{s \sharp r}} \tilde{a}_{1, m-1}\right)=-d\left\{\iota\left(X_{s}\right)\left(\bigwedge_{\varphi_{s \sharp}} \tilde{a}_{1, m-1}\right)\right\},
$$

and using Lemma 7.1, we have

$$
\begin{aligned}
\frac{d}{d s} f_{\varphi_{s}}\left(\tilde{a}_{1, l}\right)= & \sum_{m=1}^{l}(-1)^{m} d\left\{\iota\left(X_{s}\right)\left(\bigwedge_{\varphi_{s \sharp}} \tilde{a}_{1, m-1}\right)\right\} \wedge f_{\varphi_{s}}\left(a_{m}\right) \bigwedge_{r} \tilde{a}_{m+1, l} \\
& -\sum_{m=1}^{l}(-1)^{m-1} \bigwedge_{\varphi_{s \sharp}} \tilde{a}_{1, m-1} \wedge\left\{\iota\left(X_{s}\right) \varphi_{s \sharp} r\left(a_{m}\right)\right\} \bigwedge_{r} \tilde{a}_{m+1, l} \\
& +\sum_{m=1}^{l}(-1)^{m-1} \bigwedge_{\varphi_{s \sharp}} \tilde{a}_{1, m-1} \wedge I\left(\iota\left(X_{s}\right) \varphi_{s \sharp} r\left(a_{m}\right)\right) \bigwedge_{r} \tilde{a}_{m+1, l} .
\end{aligned}
$$

Computing the first term $d\{\ldots\}\left(=R H S_{1}\right)$ in the right hand side of (i), and comparing this result with the last equality, we have the desired equality. In the computation, we note that $\sum_{m=1}^{l}=\sum_{m=2}^{l}$ in $R H S_{1}$ and that the interior product is an antiderivation of degree -1 .

(ii) Using Cartan's formula for the Lie derivative, we can show (ii) by direct computation.

Lemma 7.3. The following equalities hold:

(i) $\frac{d}{d s}\left(\varphi_{s *} A-A\right)=d_{\varphi_{s *}} \beta_{s}-\iota\left(X_{s}\right) F_{\varphi_{s *} A}$ for some $\beta_{s} \in \Gamma(\operatorname{End}(T M))$, where $d_{\varphi_{s *} A}$ denotes the covariant exterior derivative with respect to $\varphi_{s *} A$.

(ii) $\frac{d}{d s} \Delta\left(\varphi_{s *} A\right) p=-d\left\{\iota\left(X_{s}\right) \Delta\left(\varphi_{s *} A\right) p\right\}$.

(iii) $\frac{d}{d s} \Delta\left(A, \varphi_{s *} A\right) p=-\iota\left(X_{s}\right) \Delta\left(\varphi_{s *} A\right) p+d R_{s}$ for some $R_{s} \in \Omega^{4 k-1}(M)$.

Proof. For (ii), since $\Delta\left(\varphi_{s *} A\right) p=\varphi_{s *} \Delta(A) p$ is a closed form, we can show the equality by using Cartan's formula.

Equalities (i) and (iii) can be obtained in the same way as the proofs of Lemma 4.2 in [Kasagawa 2008] and the equality below (5.1) in the same paper, so we omit the proofs.

For each $p \in I_{n}^{2 k}$, let

$$
\tilde{L}, \tilde{L}_{+}: H^{n-1}(M) \rightarrow \operatorname{Hom}\left(\otimes^{l} H^{1}(M), H^{n-1}(M)\right)
$$

be the homomorphisms defined as the linear extensions of

$$
\tilde{L}(w)(\tilde{a})=p(M) \cup \sum_{m=1}^{l}(-1)^{m-1}\left\langle a_{m} \cup w,[M]\right\rangle \bigcup_{j=1}^{m-1} a_{j} \cup \bigcup_{j=m+1}^{l} a_{j} / \int_{M} \operatorname{vol}
$$


and

$$
\tilde{L}_{+}(w)(\tilde{a})=-\kappa(\tilde{a}) w+\tilde{L}(w)(\tilde{a})
$$

for $\tilde{a}=a_{1} \otimes \cdots \otimes a_{l} \in \otimes^{l} H^{1}(M)$.

The following lemma can be easily checked, so we omit the proof.

Lemma 7.4. For any $w \in H^{n-1}(M), \tilde{a} \in \otimes^{l} H^{1}(M)$, and $\sigma \in \mathscr{S}_{l}$, the equalities $\tilde{L}(w)\left(\tilde{a}_{\sigma}\right)=\operatorname{sgn}(\sigma) \tilde{L}(w)(\tilde{a})$ and $\tilde{L}_{+}(w)\left(\tilde{a}_{\sigma}\right)=\operatorname{sgn}(\sigma) \tilde{L}_{+}(w)(\tilde{a})$ hold. Hence, $\tilde{L}$ and $\tilde{L}_{+}$induce the homomorphisms $L$ and $L_{+}$in (2-1) respectively.

Proposition 7.5. For any smooth curve $\left\{\varphi_{s}\right\} \subset \mathscr{D}_{\mathrm{vol}}$, the equality

$$
\frac{d}{d s} J_{\varphi_{s}}=L_{+}\left(\left[\iota\left(X_{s}\right) \mathrm{vol}\right]\right) \in \mathscr{H}
$$

holds. Here, $X_{s}$ is the time-dependent vector field on $M$ given by $d \varphi_{s} / d s=X_{s} \circ \varphi_{s}$. Proof. To begin with, we compute the derivative $d \tilde{J}_{\varphi_{s}}(\tilde{a}) / d s$ for each

$$
\tilde{a}=a_{1} \otimes \cdots \otimes a_{l} \in \otimes^{l} H^{1}(M) .
$$

By using Lemmas 7.1, 7.2, and 7.3, we have

$$
\begin{aligned}
\frac{d}{d s} \tilde{J}_{\varphi_{s}}(\tilde{a}) & \\
=\left\{\frac{d}{d s} \Delta\left(A, \varphi_{s *} A\right) p\right\} \wedge \wedge \text { r } \tilde{a} & +\left\{\frac{d}{d s} \Delta\left(\varphi_{s *} A\right) p\right\} \wedge f_{\varphi_{s}}(\tilde{a}) \\
& +\Delta\left(\varphi_{s *} A\right) p \wedge \frac{d}{d s} f_{\varphi_{s}}(\tilde{a})+\frac{d}{d s}\left\{\varphi_{s \sharp} \mu(\tilde{a})-\mu(\tilde{a})\right\} \\
=- & \kappa(\tilde{a}) \iota\left(X_{s}\right) \text { vol } \\
+\Delta\left(\varphi_{s *} A\right) p \wedge\left\{\sum_{m=1}^{l}(-1)^{m-1} \wedge_{\varphi_{s \sharp}} \tilde{a}_{1, m-1} \wedge I\left(\iota\left(X_{s}\right) \varphi_{s \sharp} r\left(a_{m}\right)\right) \wedge_{r} \tilde{a}_{m+1, l}\right\} & \left.-\iota\left(X_{s}\right) \varphi_{s \sharp} \mu(\tilde{a})\right] .
\end{aligned}
$$

Since $\varphi_{s \sharp} r\left(a_{m}\right) \wedge \mathrm{vol}=0$, we get $\left\{\iota\left(X_{s}\right) \varphi_{s \sharp} r\left(a_{m}\right)\right\}$ vol $=\varphi_{s \sharp} r\left(a_{m}\right) \wedge \iota\left(X_{s}\right)$ vol. By integrating this on $M$, we obtain

$$
I\left(\iota\left(X_{s}\right) \varphi_{s \sharp} r\left(a_{m}\right)\right) \int_{M} \mathrm{vol}=\left\langle\left[a_{m}\right] \cup\left[\iota\left(X_{s}\right) \mathrm{vol}\right],[M]\right\rangle .
$$

Using these equalities and linearity, we have

$$
d \tilde{J}_{\varphi_{s}}(\tilde{a}) / d s=\left[d J_{\varphi_{s}}(\tilde{a}) / d s\right]=\tilde{L}_{+}\left(\left[\iota\left(X_{s}\right) \operatorname{vol}\right]\right)(\tilde{a})
$$


for each $\tilde{a}$; therefore, we obtain $d J_{\varphi_{s}} / d s=\tilde{L}_{+}\left(\left[\iota\left(X_{s}\right)\right.\right.$ vol $\left.]\right)$ as homomorphisms from $\bigotimes^{l} H^{1}(M)$ to $H^{n-1}(M)$. By Lemma 7.4, we have the proposition.

Proposition 2.4 and Theorem 2.5, which are results related with volume flux homomorphisms and groups, easily follow from Proposition 7.5, so we show them and their corollaries here.

Proof of Proposition 2.4. We integrate the equality in Proposition 7.5 with respect to $s$ for $\left[\varphi_{s}\right] \in \tilde{\mathscr{D}}_{\mathrm{vol}, 0}$. Since the map $L_{+}$is linear and $J_{\mathrm{id}}=0$, we have

$$
J_{\varphi_{1}}=L_{+}\left(\int_{0}^{1}\left[\iota\left(X_{s}\right) \operatorname{vol}\right] d s\right)=L_{+}\left(\operatorname{Flux}^{\sim}\left(\left[\varphi_{s}\right]\right)\right) .
$$

This implies the proposition.

Proof of Theorem 2.5. Take $\left[\varphi_{s}\right] \in \pi^{-1}$ (id) $\subset \tilde{\mathscr{D}}_{\mathrm{vol}, 0}$. Since $\varphi_{0}=\varphi_{1}=\mathrm{id}$ and $J_{p, \text { id }}(a)=0$ for any $(p, a) \in \mathscr{P}$, by Proposition 2.4 we have $-\kappa_{p}(a) \operatorname{Flux}^{\sim}\left(\left[\varphi_{s}\right]\right)+$ $L_{p}\left(\operatorname{Flux}^{\sim}\left(\left[\varphi_{s}\right]\right)\right)(a)=0$; therefore, $\operatorname{Flux}^{\sim}\left(\left[\varphi_{s}\right]\right) \in \operatorname{ker} L_{p}(\cdot)(a)$ if $\kappa_{p}(a)=0$, otherwise Flux $\sim\left(\left[\varphi_{s}\right]\right) \in \operatorname{Im} L_{p}(\cdot)(a)$. Since the flux homomorphism is defined independently of $(p, a) \in \mathscr{P}$, the theorem follows.

Let $\operatorname{Pont}_{k}(M)$ be the space of the Pontryagin classes lying in $H^{4 k}(M)$. As an application of Theorem 2.5, we can show the following corollary:

Corollary 7.6. Let $M$ be a closed oriented smooth manifold of dimension $n$ with a volume form vol satisfying one of the following conditions:

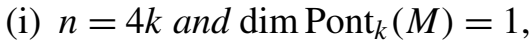

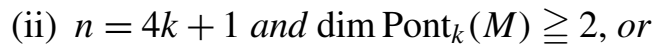

(iii) there exists a rational homology $n-1$ sphere $N \subset M$ separating $M$ into two connected submanifolds $M_{1}$ and $M_{2}$ with boundaries $N$ and $-N$ satisfying $\bigcup^{n} H^{1}\left(M_{i}, N\right)=H^{n}\left(M_{i}, N\right)$ with $i=1,2$.

Then the volume flux group of $(M, \mathrm{vol})$ is trivial; that is, $\Gamma_{\mathrm{vol}}(M)=\{0\}$.

Proof. (i) By the assumption, there is a nonzero Pontryagin class $p(M) \in H^{n}(M)$. Take $a=1 \in \wedge^{0} H^{1}(M) \cong \mathbb{R}$. The pair $(p, 1)$ belongs to $\mathscr{P} \backslash \mathscr{P}_{0}$ in Theorem 2.5. Since the map $L_{p}$ is the zero map, we have $\operatorname{Im} L_{p}(\cdot)(1)=\{0\}$. By Theorem 2.5, we have the result.

(ii) By the assumption, there are Pontryagin classes $p(M)$ and $q(M)$ which are linearly independent in $H^{n-1}(M)$. By Poincaré duality, we can take $a, b \in H^{1}(M)$ satisfying $p(M) \cup a \neq 0$ and $q(M) \cup b \neq 0$ in $H^{n}(M)$. Then the pairs $(p, a)$ and $(q, b)$ belong to $\mathscr{P} \backslash \mathscr{P}_{0}$, and we have $\operatorname{Im} L_{p}(\cdot)(a)=\mathbb{R} p(M)$ and $\operatorname{Im} L_{q}(\cdot)(b)=\mathbb{R} q(M)$. Since their intersection is $\{0\}$, the result follows. 
(iii) Let $\iota_{i}: M_{i} \hookrightarrow M$ with $i=1,2$ be the inclusions. Then we can show the induced homomorphism $\iota_{1}^{*}+\iota_{2}^{*}: H^{j}\left(M_{1}, N\right) \oplus H^{j}\left(M_{2}, N\right) \rightarrow H^{j}(M)$ is an isomorphism for each $1 \leq j \leq n-1$. By the assumption, there exist $e_{i 1}, \ldots, e_{i n} \in$ $H^{1}\left(M_{i}, N\right)$ with $\bigcup_{j=1}^{n} e_{i j} \neq 0$ in $H^{n}\left(M_{i}, N\right)$ for $i=1,2$. Put $a_{i}=\iota_{i}^{*}\left(\bigcup_{j=1}^{n} e_{i j}\right) \in$ $H^{n}(M)$ with $i=1,2$, the pairs $\left(1, a_{1}\right)$ and $\left(1, a_{2}\right)$ then belong to $\mathscr{P} \backslash \mathscr{P}_{0}$, where $p=1 \in I_{2 n}^{0}$. By the definition of the homomorphism $L=L_{p=1}$, we have $\operatorname{Im} L(\cdot)\left(a_{i}\right) \subset \iota_{i}^{*}\left(H^{n-1}\left(M_{i}, N\right)\right)$ for $i=1,2$. Using Theorem 2.5, we have $\Gamma_{\mathrm{vol}}(M) \subset \iota_{1}^{*}\left(H^{n-1}\left(M_{1}, N\right)\right) \cap \iota_{2}^{*}\left(H^{n-1}\left(M_{2}, N\right)\right)=\{0\}$.

We remark that Kędra, Kotschick, and Morita [2006] have obtained various conditions for the volume flux group $\Gamma_{\mathrm{vol}}(M)$ to vanish. (i) in Corollary 7.6 is one of them. The case of a connected sum in (iii) can also be obtained from their stronger results for volume flux groups. Moreover, in the case of (i) in Corollary 7.6, the volume flux homomorphism Flux $\sim$ descends to a homomorphism Flux: $\mathscr{D}_{\mathrm{vol}, 0} \rightarrow H^{n-1}(M) / \Gamma_{\mathrm{vol}}(M)=H^{n-1}(M)$, which is also called the volume flux homomorphism. In this case they also proved that Flux extends to a crossed homomorphism on the whole group $\mathscr{D}_{\text {vol }}$, but they didn't give an explicit formula of it.

Corollary 7.7. In the case of (i) in Corollary 7.6, take a $p \in I_{n}^{2 k}$ such that $\langle p(M),[M]\rangle=\int_{M}$ vol. Then the crossed homomorphism $J$ with respect to $p$ is rewritten as

$$
J: \mathscr{D}_{\mathrm{vol}} \rightarrow H^{n-1}(M), J_{\varphi}=\left[\Delta\left(A, \varphi_{*} A\right) p+\varphi_{*} \mu-\mu\right],
$$

and it is an extension of the flux homomorphism Flux as a crossed one.

Proof. Since $l=n-4 k=0$, we have $\wedge^{l} H^{1}(M) \cong \mathbb{R}$. Then the target of $J$ can be considered as $H^{n-1}(M)$. By the same reason, we have the simple form of $J$ as above. In particular, $\mu$ is an $(n-1)$-form satisfying $d \mu=\operatorname{vol}-\Delta(A) p$. Since $L=0$, Proposition 2.4 and $\Gamma_{\mathrm{vol}}(M)=\{0\}$ imply that the restriction of $J$ to $\mathscr{D}_{\mathrm{vol}, 0}$ is equal to Flux $\sim$ Flux.

This corollary also gives an example of the next proposition. We return to the situation of Theorem 2.3.

Proposition 7.8. If the homomorphism $L_{+}$is nontrivial, so is the cohomology class $[J]$ in Theorem 2.3.

Proof. Since $\mathscr{D}_{\mathrm{vol}, 0}$ acts trivially on the target of $J$, the restriction $\left.J\right|_{\mathscr{D}_{\mathrm{vol}, 0}}$ is a usual homomorphism. Moreover, the assumption and Proposition 2.4 imply that it is nonzero since the flux homomorphism Flux ${ }^{\sim}$ is surjective. The cohomology class

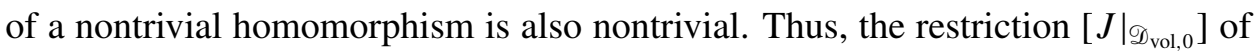
the class $[J]$ in Theorem 2.3 is nonzero; hence, so is $[J]$. 
Since the flux homomorphism Flux $\sim$ is surjective, the image $J\left(\mathscr{D}_{\mathrm{vol}, 0}\right)$ of $\mathscr{D}_{\mathrm{vol}, 0}$ by $J$ coincides with $\operatorname{Im} L_{+}$by Proposition 2.4. This implies that $J$ descends to a map

$$
\mathscr{g}: \pi_{0} \mathscr{D}_{\mathrm{vol}} \rightarrow \mathscr{H} / \operatorname{Im} L_{+} .
$$

The following lemma follows from Theorem 2.3:

Lemma 7.9. The map $\mathscr{F}$ is a crossed homomorphism and its cohomology class

$$
[\mathscr{F}] \in H^{1}\left(\pi_{0} \mathscr{D}_{\mathrm{vol}}, \mathscr{H} / \operatorname{Im} L_{+}\right)
$$

depends only on $p$, not on the choice of $A, r$ and $\mu$.

Here we give a trivial example of $J$.

Example 7.10. We consider the standard $n$-dimensional torus $T^{n}=\mathbb{R}^{n} / \mathbb{Z}^{n}$. Let $\left(x_{1}, \ldots, x_{n}\right) \in \mathbb{R}^{n}$ be the standard coordinates of $\mathbb{R}^{n}$. The volume form $d x^{1} \wedge \cdots \wedge$ $d x^{n}$ also gives the standard one vol on $T^{n}$ with $\int_{T^{n}}$ vol $=1$. One-forms $d x^{1}, \ldots, d x^{n}$ give a basis $\left[d x^{1}\right], \ldots,\left[d x^{n}\right]$ of $H^{1}\left(T^{n}\right)$. Put $Y=\left[d x^{1}\right] \wedge \cdots \wedge\left[d x^{n}\right]$. Then this is the base of $\bigwedge^{n} H^{1}\left(T^{n}\right)$.

We consider the case of $p=1$; hence, $k=0, l=n-4 k=n$, and a connection $A$ is not needed. We take the section $r: H^{1}\left(T^{n}\right) \rightarrow Z^{1}\left(T^{n}\right)$ given by $r\left(\left[d x^{i}\right]\right)=d x^{i}$ with $i=1, \ldots, n$. Since $\kappa(Y)$ vol $-\bigwedge_{r} Y=0$, we can take $\mu=0$. Thus, we have the crossed homomorphism $J: \mathscr{D}_{\mathrm{vol}} \rightarrow \mathscr{H}=\operatorname{Hom}\left(\bigwedge^{n} H^{1}\left(T^{n}\right), H^{n-1}\left(T^{n}\right)\right)$. In this case we have $J_{\varphi}(a)=\left[f_{\varphi}(a)\right]$ because $\mu=0$ and $p=1$. Since $\kappa(Y)=1$ and $L(w)(Y)=w$ for any $w \in H^{n-1}\left(T^{n}\right)$, which are easily checked, we have $J_{\varphi}(Y)=0$ for any $\varphi \in \mathscr{D}_{\mathrm{vol}, 0}$ by Proposition 2.4. Let $\varphi \in \mathrm{SL}(n, \mathbb{Z}) \subset \mathscr{D}_{\mathrm{vol}}$. We have $\varphi_{\sharp} r(a)-r(a)=0$ for all $a \in H^{1}\left(T^{n}\right)$. This implies $f_{\varphi}(a)=0$ hence $J_{\varphi}=0$. Thus, $J$ descends to a crossed homomorphism $\mathscr{F}^{\sim}: \pi_{0} \mathscr{D}_{\mathrm{vol}} \rightarrow \mathscr{H}$, whose image of $\operatorname{SL}(n, \mathbb{Z})\left(\subset \pi_{0} \mathscr{D}_{\mathrm{vol}}\right)$ is $\{0\}$.

Let $n \geqq 5$. There is a split exact sequence $0 \rightarrow K \rightarrow \pi_{0} \operatorname{Diff}\left(T^{n}\right) \rightarrow \operatorname{GL}(n, \mathbb{Z}) \rightarrow 0$, where $K=\mathbb{Z}_{2}^{\infty} \oplus\left(\begin{array}{l}n \\ 2\end{array}\right) \mathbb{Z}_{2} \oplus \sum_{i=0}^{n}\left(\begin{array}{c}n \\ i\end{array}\right) \Gamma_{i+1}$, by [Hatcher 1978] for $n \geqq 5$ and [Hsiang and Sharpe 1976] for $n \geqq 6$. So we have a split one $0 \rightarrow K \rightarrow \pi_{0} \mathscr{D}_{\mathrm{vol}} \rightarrow \operatorname{SL}(n, \mathbb{Z}) \rightarrow 0$. Here the action of $K$ on $H^{*}\left(T^{n}\right)$ is trivial. The groups $\Gamma_{i+1}$ of twisted spheres are finite abelian groups (see [Milnor 2011] and its references). Hence, every element of $K$ is of finite order. This shows $\mathscr{F}^{\sim}(K)=\{0\}$; hence, $\operatorname{Im} \mathscr{F}^{\sim}=\{0\}$, so we have $J=0$ for the choice of $r$ and $\mu$ as above. Its cohomology class [ $J]$ is also zero.

\section{Proof of Theorem 2.6}

In this section we will prove Theorem 2.6, whose main part is that the crossed homomorphism $\mathscr{F}$ is essentially independent of the choice of volume form on $M$. 
Let vol and vol' be two positive volume forms on $M$. By Moser [1965], there exist $\epsilon>0$ and $\xi \in \operatorname{Diff}_{+}(M)$ such that $\xi_{*} \operatorname{vol}=\epsilon \operatorname{vol}^{\prime}$. We consider the isomorphism

$$
c_{\xi}: \mathscr{D}_{\mathrm{vol}} \rightarrow \mathscr{D}_{\mathrm{vol}^{\prime}} \quad \text { with } \quad c_{\xi}(\varphi)=\xi \varphi \xi^{-1}
$$

given by the conjugation by $\xi$. Let $C_{0}^{\infty}(M)^{\prime}$ be the vector space of smooth functions on $M$ with integral 0 with respect to vol' . It is easy to see that the map $\xi_{*}: C_{0}^{\infty}(M) \rightarrow$ $C_{0}^{\infty}(M)^{\prime}$ given by $\xi_{*} h:=\left(\xi^{-1}\right)^{*} h$ for $h \in C_{0}^{\infty}(M)$ is a well-defined isomorphism. Let

$$
\xi_{\sharp}: \operatorname{Hom}\left(H^{1}(M), C_{0}^{\infty}(M)\right) \rightarrow \operatorname{Hom}\left(H^{1}(M), C_{0}^{\infty}(M)^{\prime}\right)
$$

be the homomorphism defined by $\left(\xi_{\sharp} h\right)(a)=\xi_{*}\left(h\left(\xi^{*}(a)\right)\right)$ for $a \in H^{1}(M)$ and $h \in \operatorname{Hom}\left(H^{1}(M), C_{0}^{\infty}(M)\right)$. We also need the homomorphism

$$
\xi_{\sharp}: \mathscr{H} \rightarrow \mathscr{H}
$$

defined in the same way as before. Here we use the same symbol $\xi_{\sharp}$ as above.

We recall that $r: H^{1}(M) \rightarrow Z^{1}(M)$ is an injective linear section of the projection $Z^{1}(M) \rightarrow H^{1}(M)$ and so is $\xi_{\sharp} r$. Let $f$ be the crossed homomorphism in Lemma 2.1 with respect to the volume form vol and $r$, and $f^{\prime}$ with respect to vol' and $\xi_{\sharp} r$. Let $J$ be the crossed homomorphism in Theorem 2.3 with respect to volume forms vol and $S=\left\{p, A, r, \mu\left(=\mu_{S}\right)\right\}$, and $J^{\prime}$ with respect to $\operatorname{vol}^{\prime}$ and $S^{\prime}=\left\{p, \xi_{*} A, \xi_{\sharp} r, \xi_{\sharp} \mu\right\}$.

Lemma 8.1. The following diagrams commute:

(i)

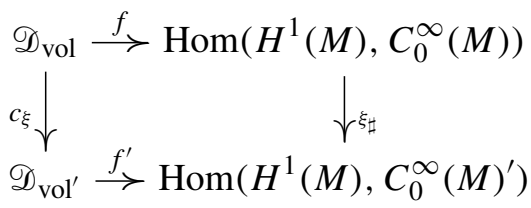

and

(ii)

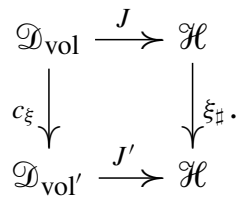

Proof. Let $\varphi \in \mathscr{D}_{\mathrm{vol}}$.

(i) For any $a \in H^{1}(M)$, we have

$d\left\{\left(\xi_{\sharp} f_{\varphi}\right)(a)\right\}=\left\{\xi_{\sharp} \varphi_{\sharp} r\right\}(a)-\left(\xi_{\sharp} r\right)(a)=\left\{c_{\xi}(\varphi)_{\sharp} \xi_{\sharp} r\right\}(a)-\left(\xi_{\sharp} r\right)(a)=d\left\{f_{c_{\xi}(\varphi)}^{\prime}(a)\right\} ;$ then $\left(\xi_{\sharp} f_{\varphi}\right)(a)=f_{c_{\xi}(\varphi)}^{\prime}(a)$ because $\left(\xi_{\sharp} f_{\varphi}\right)(a), f_{c_{\xi}(\varphi)}^{\prime}(a) \in C_{0}^{\infty}(M)^{\prime}$. This implies (i). (ii) For any $a \in \bigwedge^{l} H^{1}(M)$, we can show

$$
d\left\{\xi_{\sharp} \mu(a)\right\}=\kappa^{\prime}(a) \operatorname{vol}^{\prime}-\Delta\left(\xi_{*} A\right) p \wedge \bigwedge_{\xi_{\sharp} r} a,
$$

where $\kappa^{\prime}$ is the $\kappa$ with respect to $\mathrm{vol}^{\prime}$. Thus, we can take $\xi_{\sharp} \mu$ as $\mu$ in Lemma 2.2 with respect to $\operatorname{vol}^{\prime}$ and $\left\{p, \xi_{*} A, \xi_{\sharp} r\right\}$. Using (i), we can find $\left(\xi_{\sharp} J_{\varphi}\right)(a)=J_{c_{\xi}(\varphi)}^{\prime}(a)$ by direct computation. This implies that the diagram commutes.

Let $L_{+}, L_{+}^{\prime}: H^{n-1}(M) \rightarrow \mathscr{H}$ be the homomorphisms in (2-1) with respect to vol and vol' respectively. 
Lemma 8.2. (i) $\operatorname{Im} L_{+}=\operatorname{Im} L_{+}^{\prime}$.

(ii) $\xi_{\sharp}\left(\operatorname{Im} L_{+}\right)=\operatorname{Im} L_{+}^{\prime}$.

Proof. Since $\xi_{*} \mathrm{vol}=\epsilon \mathrm{vol}^{\prime}$, we have $\int_{M} \mathrm{vol}=\epsilon \int_{M} \mathrm{vol}^{\prime}$ and $\kappa^{\prime}=\epsilon \kappa$. Those imply $L_{+}^{\prime}=\epsilon L_{+}$and $L_{+}^{\prime}\left(\xi_{*} w\right)=\epsilon \xi_{\sharp}\left\{L_{+}(w)\right\}$ for all $w \in H^{n-1}(M)$, which show (i) and (ii).

By Lemmas 8.1 and 8.2, we have the following commutative diagram:

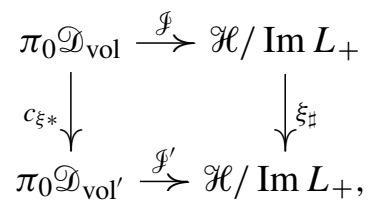

where $\mathscr{g}$ and $\mathscr{g}^{\prime}$ are induced from $J$ and $J^{\prime}$ respectively. By Moser, the inclusion $\mathscr{D}_{\mathrm{vol}} \hookrightarrow \mathscr{D}$ is a weak homotopy equivalence. Hence, it induces an isomorphism $\pi_{0} \mathscr{D}_{\mathrm{vol}} \cong \pi_{0} \mathscr{D}$.

Hereafter, we assume that $\xi$ is isotopic to the identity $\xi \simeq \mathrm{id}$, which is always possible in Moser's method. We have the following commutative diagram:

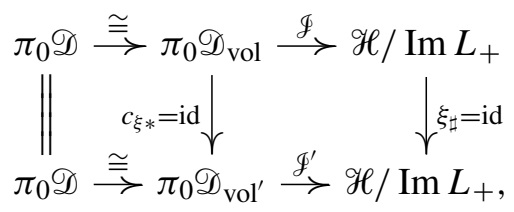

which implies that any $\mathscr{F}$ with respect to vol coincides with some $\mathscr{F}^{\prime}$ with respect to vol'. The remaining part of Theorem 2.6 can be shown in the same way as Theorem 2.3. Thus, we complete the proof of Theorem 2.6.

\section{Johnson homomorphisms}

Let $\Sigma_{g}$ be a closed oriented surface of genus $g \geqq 3$, and $H_{\mathbb{Z}}:=H_{1}\left(\Sigma_{g} ; \mathbb{Z}\right)$ its first homology group with coefficients in $\mathbb{Z}$. Let Diff ${ }_{+}\left(\Sigma_{g}\right)$ be the group of orientation preserving diffeomorphisms of $\Sigma_{g}$ with the $C^{\infty}$ topology. The mapping class group $\mathcal{M}_{g}$ of $\Sigma_{g}$ is the group of path components of $\operatorname{Diff}_{+}\left(\Sigma_{g}\right)$. The standard action of $\mu_{g}$ on $H_{\mathbb{Z}}$ induces a well-known representation $M_{g} \rightarrow \operatorname{Aut}\left(H_{\mathbb{Z}}, \cdot\right) \cong \operatorname{Sp}(2 g, \mathbb{Z})$, where $\cdot$ denotes the intersection pairing on $H_{\mathbb{Z}}$. The kernel $\rrbracket_{g}$ of the representation is called the Torelli group. Take a base point $* \in \Sigma_{g}$ as depicted in Figure 1, and fix it. We can consider the mapping class group $\mu_{g, *}$ of $\left(\Sigma_{g}, *\right)$, which is the group of path components of the subgroup $\operatorname{Diff}_{+}\left(\Sigma_{g}, *\right) \subset \operatorname{Diff}_{+}\left(\Sigma_{g}\right)$ of diffeomorphisms preserving the base point. The kernel $\Phi_{g, *}$ of the composition $\mu_{g, *} \rightarrow M_{g} \rightarrow \operatorname{Sp}(2 g, \mathbb{Z})$ is also called the Torelli group. 


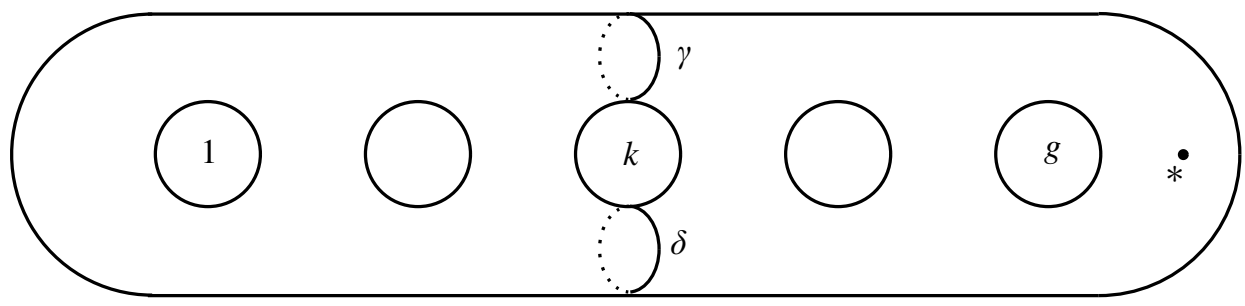

Figure 1. A base point $*$ and a bounding pair $(\gamma, \delta)$.

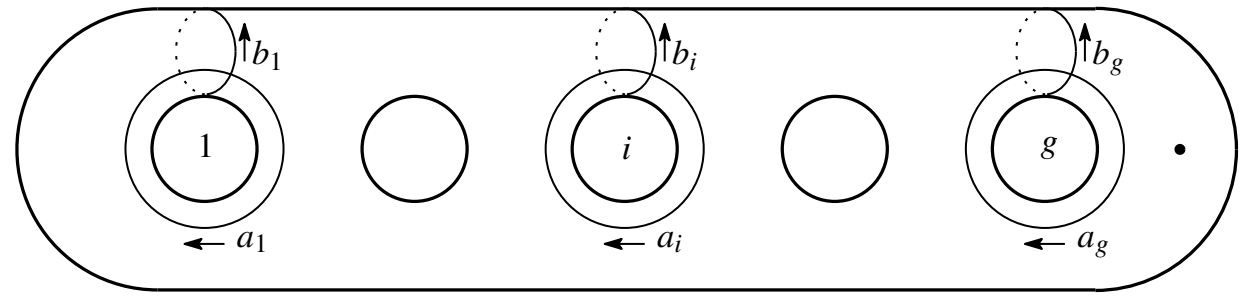

Figure 2. A basis $a_{i}, b_{i}$ of $H_{\mathbb{Z}}$.

Let $T_{\gamma}$ be a Dehn twist along a simple closed curve (SCC) $\gamma$ in $\Sigma_{g}$. Let $(\gamma, \delta)$ be a bounding pair (BP); that is, a pair of disjoint homologous SCC's which are not homologically trivial. A BP map is given by $T_{\gamma} T_{\delta}^{-1}$. D. Johnson [1979] showed that $\mathscr{I}_{g, *}$ are generated by all BP maps. He also defined the (first) Johnson homomorphism $\tau=\tau_{g}: \mathscr{I}_{g, *} \rightarrow \bigwedge^{3} H_{\mathbb{Z}}$. Let

$$
\varphi_{k}=T_{\gamma} T_{\delta}^{-1} \in \operatorname{Diff}_{+}\left(\Sigma_{g}, *\right)
$$

be the BP map for the BP $(\gamma, \delta)$ as depicted in Figure 1. Johnson [1980] calculated the value of $\tau$ at $\varphi_{k} \in \mathcal{M}_{g, *}$, which is $\tau\left(\varphi_{k}\right)=\left(\sum_{i=1}^{k-1} a_{i} \wedge b_{i}\right) \wedge b_{k}$. Here $\left\{a_{i}, b_{i}\right\}_{i=1}^{g}$ is the symplectic basis for $H_{\mathbb{Z}}$ as depicted in Figure 2, and the mapping class $\left[\varphi_{k}\right]$ of $\varphi_{k}$ is also denoted by the same symbol $\varphi_{k}$. Hereafter we use this symbol for a diffeomorphism and its mapping class.

The Johnson homomorphism $\tau$ descends to $\tau: \Phi_{g} \rightarrow \bigwedge^{3} H_{\mathbb{Z}} / H_{\mathbb{Z}}$, which is also a Johnson homomorphism, and is denoted by the same letter $\tau$. Here, $H_{\mathbb{Z}}$ is considered a subgroup of $\wedge^{3} H_{\mathbb{Z}}$ by the injection $H_{\mathbb{Z}} \ni x \mapsto x \wedge\left(\sum_{i=1}^{g} a_{i} \wedge b_{i}\right) \in \wedge^{3} H_{\mathbb{Z}}$. Let $\left\{a_{i}^{*}, b_{i}^{*}\right\}$ be the dual basis of $H^{1}\left(\Sigma_{g} ; \mathbb{Z}\right)=H_{\mathbb{Z}}^{*}$ to $\left\{a_{i}, b_{i}\right\}$. Poincaré duality gives the identification $H_{\mathbb{Z}} \cong H_{\mathbb{Z}}^{*}$ by $a_{i} \mapsto-b_{i}^{*}, b_{i} \mapsto a_{i}^{*}$. Using it, we have

$$
\bigwedge^{3} H_{\mathbb{Z}} \subset H_{\mathbb{Z}} \otimes \bigwedge^{2} H_{\mathbb{Z}} \cong \operatorname{Hom}\left(H_{\mathbb{Z}}, \bigwedge^{2} H_{\mathbb{Z}}\right) \text {. }
$$

The image $\tau\left(\varphi_{k}\right)=\left(\sum_{i=1}^{k-1} a_{i} \wedge b_{i}\right) \wedge b_{k} \in \wedge^{3} H_{\mathbb{Z}}$ of $\varphi_{k}$ by $\tau$ is given, as an element 
of $\operatorname{Hom}\left(H_{\mathbb{Z}} \bigwedge^{2} H_{\mathbb{Z}}\right)$, by

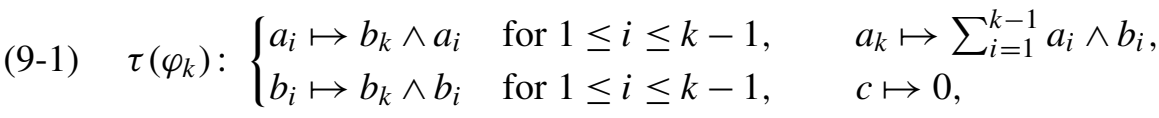

where $c$ denotes the remaining base elements, and it is given as an element of $H_{\mathbb{Z}}^{*} \otimes \wedge^{2} H_{\mathbb{Z}}$ by

$$
\tau\left(\varphi_{k}\right)=-\sum_{i=1}^{k-1}\left\{a_{i}^{*} \otimes\left(a_{i} \wedge b_{k}\right)+b_{i}^{*} \otimes\left(b_{i} \wedge b_{k}\right)-a_{k}^{*} \otimes\left(a_{i} \wedge b_{i}\right)\right\} .
$$

\section{The homomorphism $L_{+}$in the 2-dimensional case}

In this section we will compute the homomorphism $L_{+}$defined in Section 2 in the 2-dimensional case with $p=1$.

Let $M=\Sigma_{g}$ be a closed oriented surface of genus $g \geqq 3$ and $\omega$ an area form with area $A=\int_{\Sigma_{g}} \omega$. Let $\left\{a_{i}, b_{i}\right\}$ and $\left\{a_{i}^{*}, b_{i}^{*}\right\}$ be the dual bases of $H_{\mathbb{Z}}$ and $H_{\mathbb{Z}}^{*}$ to each other in Section 9. Set $H=H_{1}\left(\Sigma_{g}\right)$ and $H^{*}=H^{1}\left(\Sigma_{g}\right)$. The bases $\left\{a_{i}, b_{i}\right\}$ and $\left\{a_{i}^{*}, b_{i}^{*}\right\}$ can also be considered bases of $H$ and $H^{*}$ respectively.

In this case, since $n=l=2$ and $p=1$, the homomorphism $L_{+}: H^{*} \rightarrow$ $\operatorname{Hom}\left(\bigwedge^{2} H^{*}, H^{*}\right)$ of $(2-1)$ is given by

$$
L_{+}(w)=\left[a \mapsto \frac{1}{A}\left\{\left\langle c_{1} \cup w,\left[\Sigma_{g}\right]\right\rangle c_{2}-\left\langle c_{2} \cup w,\left[\Sigma_{g}\right]\right\rangle c_{1}-\left\langle c_{1} \cup c_{2},\left[\Sigma_{g}\right]\right\rangle w\right\}\right]
$$

for $w \in H^{*}$ and $c=c_{1} \wedge c_{2} \in \wedge^{2} H^{*}$. In particular, for $w=a_{l}^{*}, b_{l}^{*} \in H^{*}$ with $1 \leq l \leq g$, we have

and

$$
L_{+}\left(a_{l}^{*}\right):\left\{\begin{array}{l}
a_{i}^{*} \wedge a_{j}^{*} \mapsto 0, \\
b_{i}^{*} \wedge b_{j}^{*} \mapsto \frac{1}{A}\left(-\delta_{i l} b_{j}^{*}+b_{i}^{*} \delta_{j l}\right), \\
a_{i}^{*} \wedge b_{j}^{*} \mapsto \frac{1}{A}\left(a_{i}^{*} \delta_{j l}-\delta_{i j} a_{l}^{*}\right),
\end{array}\right.
$$

$$
L_{+}\left(b_{l}^{*}\right):\left\{\begin{array}{l}
a_{i}^{*} \wedge a_{j}^{*} \mapsto \frac{1}{A}\left(\delta_{i l} a_{j}^{*}-a_{i}^{*} \delta_{j l}\right), \\
b_{i}^{*} \wedge b_{j}^{*} \mapsto 0, \\
a_{i}^{*} \wedge b_{j}^{*} \mapsto \frac{1}{A}\left(\delta_{i l} b_{j}^{*}-\delta_{i j} b_{l}^{*}\right),
\end{array}\right.
$$

for all $1 \leq i, j \leq g$, where $\delta_{i j}$ denotes Kronecker's delta.

We can represent $L_{+}\left(a_{l}^{*}\right)$ and $L_{+}\left(b_{l}^{*}\right)$ as elements of $H^{*} \otimes \bigwedge^{2} H$ as follows:

$$
\begin{aligned}
& L_{+}\left(a_{l}^{*}\right)=\frac{1}{2 A} \sum_{i=1}^{g}\left\{a_{i}^{*} \otimes\left(a_{i} \wedge b_{l}\right)+b_{i}^{*} \otimes\left(b_{i} \wedge b_{l}\right)-a_{l}^{*} \otimes\left(a_{i} \wedge b_{i}\right)\right\}, \\
& L_{+}\left(b_{l}^{*}\right)=\frac{1}{2 A} \sum_{j=1}^{g}\left\{a_{j}^{*} \otimes\left(a_{l} \wedge a_{j}\right)+b_{j}^{*} \otimes\left(a_{l} \wedge b_{j}\right)-b_{l}^{*} \otimes\left(a_{j} \wedge b_{j}\right)\right\} .
\end{aligned}
$$


We remark that under the identification $\left(\wedge^{2} H^{*}\right)^{*} \cong \bigwedge^{2} H$, the dual basis of $\bigwedge^{2} H^{*}$ to $\left\{a_{i}^{*} \wedge a_{j}^{*}(i<j), b_{i}^{*} \wedge b_{j}^{*}(i<j), a_{i}^{*} \wedge b_{j}^{*}(\forall i, j)\right\}$ is given by

$$
\left\{\frac{1}{2} a_{i} \wedge a_{j}(i<j), \frac{1}{2} b_{i} \wedge b_{j}(i<j), \frac{1}{2} a_{i} \wedge b_{j}(\forall i, j)\right\} \subset \wedge^{2} H
$$

by our convention.

\section{A section $r$}

In this section we will explicitly give a section $r$ as in Section 2, which is needed in order to define our crossed homomorphism.

Let $T_{i}(1 \leq i \leq g)$ be compact submanifolds of $\Sigma_{g}$ - as depicted in Figure 3 which are diffeomorphic to a 2-torus with two open disks deleted. We consider each $T_{i}$ as a submanifold of $\mathbb{R}^{2} /(2 \pi \mathbb{Z})^{2}$ and use the induced coordinates $(x, y) \in T_{i} \subset$ $\mathbb{R}^{2} /(2 \pi \mathbb{Z})^{2}$. But we mainly take $(x, y) \in(-\pi, \pi] \times(-\pi, \pi] \backslash$ (int $D^{2} \amalg$ int $\left.D^{2}\right) \subset T_{i}$ as depicted in Figure 4. We assume

$(-\pi, \pi] \times[-1,1] \cup[-1,1] \times(-\pi, \pi] \subset(-\pi, \pi] \times(-\pi, \pi] \backslash$ (int $D^{2} \amalg$ int $\left.D^{2}\right)$.

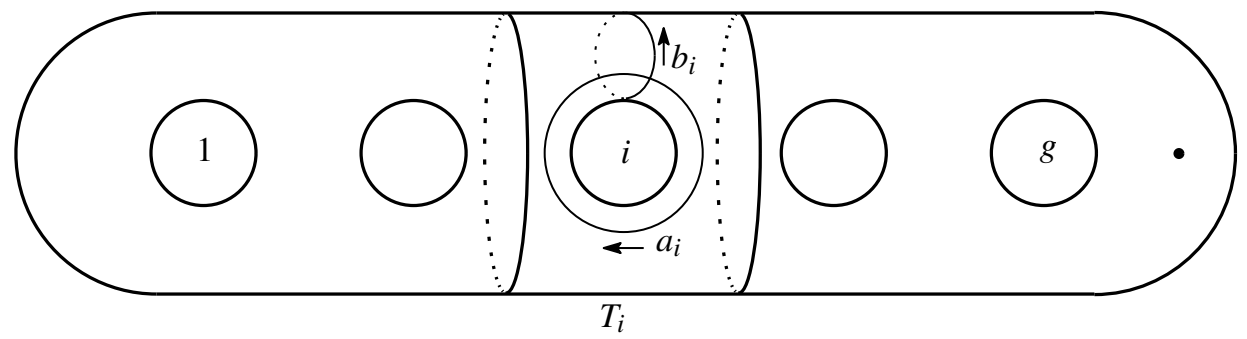

Figure 3. Compact submanifolds $T_{i}$ of $\Sigma_{g}$.
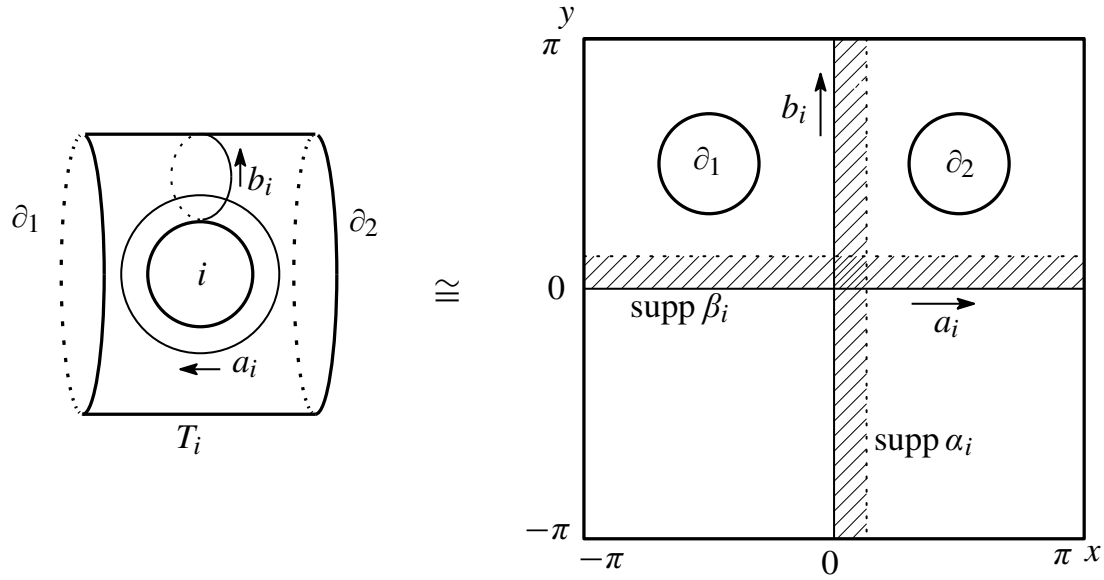

Figure 4. Coordinates of $T_{i}$. 
Let $\rho: \mathbb{R} \rightarrow \mathbb{R}$ be a smooth function such that

$$
\rho(x)= \begin{cases}0 & \text { if } x \leq 0 \\ \text { monotone increasing } & \text { if } 0<x<\varepsilon \\ 1 & \text { if } \varepsilon \leq x\end{cases}
$$

for a sufficiently small $\varepsilon>0$. We define closed 1 -forms $\alpha_{i}, \beta_{i} \in Z^{1}\left(\Sigma_{g}\right)$ with $1 \leq i \leq g$ by

$$
\alpha_{i}(p)=\left\{\begin{array}{ll}
d \rho(x) & \text { if } p=(x, y) \in T_{i}, \\
0 & \text { if } p \in \Sigma_{g} \backslash T_{i},
\end{array} \quad \beta_{i}(p)= \begin{cases}d \rho(y) & \text { if } p=(x, y) \in T_{i}, \\
0 & \text { if } p \in \Sigma_{g} \backslash T_{i}, .\end{cases}\right.
$$

It is easy to see that $\left\{\alpha_{i}, \beta_{i}\right\}$ represents the basis $\left\{a_{i}^{*}, b_{i}^{*}\right\}$ of $H^{*}$.

We use the section $r: H^{*} \rightarrow Z^{1}\left(\Sigma_{g}\right)$ of the projection $Z^{1}\left(\Sigma_{g}\right) \rightarrow H^{*}$ defined as the linear extension of $r\left(a_{i}^{*}\right)=\alpha_{i}$ and $r\left(b_{i}^{*}\right)=\beta_{i}$ with $1 \leq i \leq g$.

\section{BP maps $\varphi_{k}$}

In this section, we will define BP maps $\varphi_{k}$ with $1<k<g$ as $\omega$-preserving diffeomorphisms. We will compute $\mathscr{F}_{\varphi_{k}}$ in later sections.

Let $(x, y) \in T_{k}$ be the local coordinates of $T_{k}$ given in Section 11 . We explicitly give simple closed curves $\gamma$ and $\delta$ on $T_{k}$ by

$$
\left\{\left(-\frac{5}{2} \varepsilon, y\right) \in T_{k} \mid y \in \mathbb{R} / 2 \pi \mathbb{Z}\right\}
$$

and

$$
\left\{\left(\pi-\frac{5}{2} \varepsilon, y\right) \in T_{k} \mid y \in \mathbb{R} / 2 \pi \mathbb{Z}\right\}
$$

respectively. A BP map $\varphi_{k}: \Sigma_{g} \rightarrow \Sigma_{g}$ is given by

$\varphi_{k}(p)= \begin{cases}(x, a(x)+y) & \text { if } p=(x, y) \in[-3 \varepsilon,-2 \varepsilon] \times(-\pi, \pi] \subset T_{k} \\ (x,-a(x-\pi)+y) & \text { if } p=(x, y) \in[\pi-3 \varepsilon, \pi-2 \varepsilon] \times(-\pi, \pi] \subset T_{k} \\ p & \text { otherwise, }\end{cases}$

where $a:[-3 \varepsilon,-2 \varepsilon] \rightarrow \mathbb{R}$ is a nonincreasing smooth function satisfying

$$
a(t)= \begin{cases}0 & \text { near } t=-3 \varepsilon \\ -2 \pi & \text { near } t=-2 \varepsilon .\end{cases}
$$

See Figure 5 for the support of $\varphi_{k}$. It is easy to check $\varphi_{k} \in \mathscr{D}_{\omega}=\left\{\varphi \in \operatorname{Diff}\left(\Sigma_{g}\right) \mid\right.$ $\left.\varphi^{*} \omega=\omega\right\}$ and

$\varphi_{k}^{-1}(p)= \begin{cases}(x,-a(x)+y) & \text { if } p=(x, y) \in[-3 \varepsilon,-2 \varepsilon] \times(-\pi, \pi] \subset T_{k}, \\ (x, a(x-\pi)+y) & \text { if } p=(x, y) \in[\pi-3 \varepsilon, \pi-2 \varepsilon] \times(-\pi, \pi] \subset T_{k}, \\ p & \text { otherwise. }\end{cases}$ 


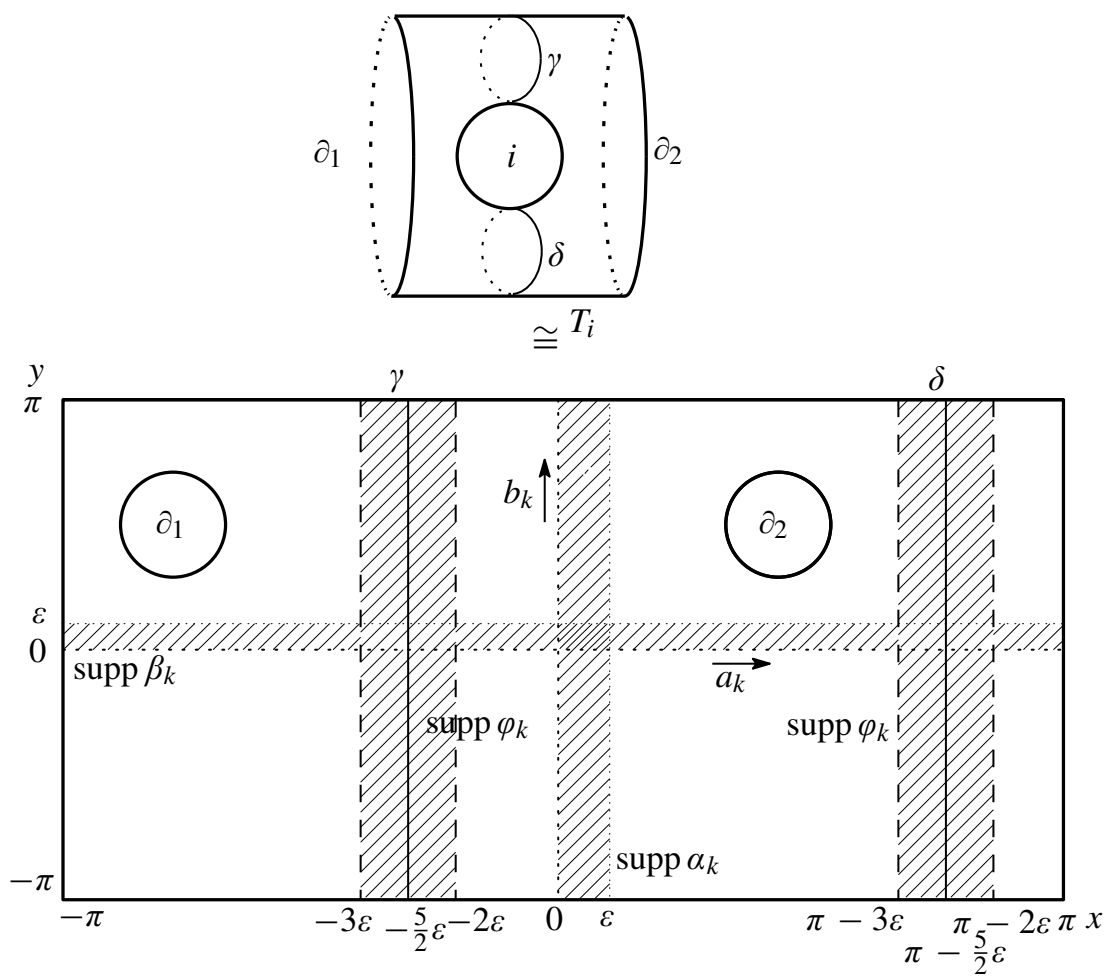

Figure 5. Simple closed curves $\gamma$ and $\delta$ and $\operatorname{supp} \varphi_{k}$.

\section{3. $J_{\varphi_{k}}$}

In this section we will compute $J_{\varphi_{k}}$ and prove Theorem 2.7 without showing some lemmas, whose proofs are given in the next section.

In the case of $(M, \mathrm{vol})=\left(\Sigma_{g}, \omega\right)$, the crossed homomorphism $J$ is written as $J: \mathscr{D}_{\omega} \ni \varphi \mapsto J_{\varphi} \in \mathscr{H}=\operatorname{Hom}\left(\bigwedge^{2} H^{*}, H^{*}\right)$, where $J_{\varphi}(a)=\left[\tilde{J}_{\varphi}(a)\right] \in H^{*}$ and

$$
\tilde{J}_{\varphi}(a)=f_{\varphi}\left(c_{1}\right) r\left(c_{2}\right)-\varphi_{\sharp} r\left(c_{1}\right) \cdot f_{\varphi}\left(c_{2}\right)+\varphi_{\sharp} \mu(a)-\mu(a)
$$

for $a=c_{1} \wedge c_{2} \in \bigwedge^{2} H^{*}$.

Hereafter, we fix $k$ as $1<k<g$ and write $\varphi=\varphi_{k}$ for simplicity.

Let $* \in \Sigma_{g}$ be the base point depicted in Figure 1 as before. Since $\varphi_{*} \beta_{k}-\beta_{k}$ is exact, we have a unique function $h \in C^{\infty}\left(\Sigma_{g}\right)$ satisfying $d h=\varphi_{*} \beta_{k}-\beta_{k}$ and $h(*)=0$. Let $\Sigma_{k-}$ and $\Sigma_{k+}$ be the connected components of $\Sigma_{g} \backslash T_{k}$ such that $* \in \Sigma_{k+}$. The following lemma is easily checked, so its proof is omitted:

Lemma 13.1. (i) $h \equiv-1$ on $\Sigma_{k-}$.

(ii) $h \equiv 0$ on $\Sigma_{k+}$.

Set $h_{0}:=\int_{\Sigma_{g}} h \omega$. 
Lemma 13.2. Assume $\varphi=\varphi_{k}$ with $1<k<g$.

(i) $J_{\varphi}(a)=0$ for $a=a_{i}^{*} \wedge a_{j}^{*}(\forall i, j)$ or $a=b_{i}^{*} \wedge b_{j}^{*}(i \neq k, j \neq k)$ or $a=a_{i}^{*} \wedge b_{j}^{*}$ $(i \neq j, i \neq k, j \neq k)$.

(ii) For $a=b_{i}^{*} \wedge b_{k}^{*}(i \neq k)$, we have $J_{\varphi}(a)= \begin{cases}\left(1+h_{0} / A\right) b_{i}^{*} & \text { if } i<k, \\ \left(h_{0} / A\right) b_{i}^{*} & \text { if } i>k .\end{cases}$

(iii) For $a=a_{i}^{*} \wedge b_{k}^{*}(i \neq k)$, we have $J_{\varphi}(a)= \begin{cases}\left(1+h_{0} / A\right) a_{i}^{*} & \text { if } i<k, \\ \left(h_{0} / A\right) a_{i}^{*} & \text { if } i>k,\end{cases}$

(iv) For $a=a_{k}^{*} \wedge b_{j}^{*}$ with $j \neq k$, we have $J_{\varphi}(a)=0$.

(v) For $a=a_{i}^{*} \wedge b_{i}^{*}$, we have $J_{\varphi}(a)= \begin{cases}-\left(1+h_{0} / A\right) a_{k}^{*} & \text { if } i<k, \\ 0 & \text { if } i=k, \\ -\left(h_{0} / A\right) a_{k}^{*} & \text { if } i>k,\end{cases}$

For a while we admit this lemma. It implies that $J_{\varphi}$ is given by

$$
\begin{aligned}
J_{\varphi}= & \sum_{i<k}\left(b_{i}^{*} \wedge b_{k}^{*}\right)^{*} \otimes\left(1+\frac{h_{0}}{A}\right) b_{i}^{*}+\sum_{i>k}\left(b_{i}^{*} \wedge b_{k}^{*}\right)^{*} \otimes \frac{h_{0}}{A} b_{i}^{*} \\
& +\sum_{i<k}\left(a_{i}^{*} \wedge b_{k}^{*}\right)^{*} \otimes\left(1+\frac{h_{0}}{A}\right) a_{i}^{*}+\sum_{i>k}\left(a_{i}^{*} \wedge b_{k}^{*}\right)^{*} \otimes \frac{h_{0}}{A} a_{i}^{*} \\
& +\sum_{i<k}\left(a_{i}^{*} \wedge b_{i}^{*}\right)^{*} \otimes\left(-1-\frac{h_{0}}{A}\right) a_{k}^{*}+\sum_{i>k}\left(a_{i}^{*} \wedge b_{i}^{*}\right)^{*} \otimes\left(-\frac{h_{0}}{A}\right) a_{k}^{*}
\end{aligned}
$$

as an element of $\left(\bigwedge^{2} H^{*}\right)^{*} \otimes H^{*}$. Under the identification $\left(\bigwedge^{2} H^{*}\right)^{*} \cong \bigwedge^{2} H$, which is given by $\left(a_{i}^{*} \wedge a_{j}^{*}\right)^{*} \mapsto \frac{1}{2} a_{i} \wedge a_{j},\left(b_{i}^{*} \wedge b_{j}^{*}\right)^{*} \mapsto \frac{1}{2} b_{i} \wedge b_{j}$, and $\left(a_{i}^{*} \wedge b_{j}^{*}\right)^{*} \mapsto \frac{1}{2} a_{i} \wedge b_{j}$ by our convention as remarked before, we have

$$
\begin{aligned}
J_{\varphi}= & \frac{1}{2} \sum_{i<k}\left\{b_{i}^{*} \otimes\left(b_{i} \wedge b_{k}\right)+a_{i}^{*} \otimes\left(a_{i} \wedge b_{k}\right)-a_{k}^{*} \otimes\left(a_{i} \wedge b_{i}\right)\right\} \\
& +\frac{h_{0}}{2 A} \sum_{i=1}^{g}\left\{b_{i}^{*} \otimes\left(b_{i} \wedge b_{k}\right)+a_{i}^{*} \otimes\left(a_{i} \wedge b_{k}\right)-a_{k}^{*} \otimes\left(a_{i} \wedge b_{i}\right)\right\}
\end{aligned}
$$

as an element of $H^{*} \otimes \bigwedge^{2} H$; therefore, by (9-2) and (10-1), we obtain

$$
J_{\varphi}=-\frac{1}{2} \tau(\varphi)+h_{0} L_{+}\left(a_{k}^{*}\right) .
$$

Proof of Theorem 2.7. As mentioned before, we use the same symbol $\varphi$ for the mapping class $[\varphi] \in \pi_{0} \mathscr{D} \cong \pi_{0} \mathscr{D}_{\omega}$ of $\varphi \in \mathscr{D}_{\omega}$. The computation above implies $\mathscr{F}_{\varphi}=$ $-\frac{1}{2} j \circ \tau(\varphi)$ for $\varphi=\varphi_{k}(1<\forall k<g)$, where $j$ is the homomorphism (2-2). Johnson [1980] showed that $\tau$ is $\pi_{0} \mathscr{D}$-equivariant, which means $\tau\left(\psi \varphi \psi^{-1}\right)=\psi_{*}\{\tau(\varphi)\}$ for any $\varphi \in \mathscr{I}_{g}$ and $\psi \in \pi_{0} \mathscr{D}$. $\mathscr{F}$ is also $\pi_{0} \mathscr{D}$-equivariant on $\mathscr{I}_{g}$; in fact, since $\mathscr{\mathscr { F }}$ is a crossed homomorphism on $\pi_{0} \mathscr{D}$, we have $\mathscr{E}_{\psi \varphi \psi^{-1}}=\mathscr{F}_{\psi}+\psi_{\sharp} \mathscr{F}_{\varphi}-\left(\psi \varphi \psi^{-1}\right)_{\sharp} \mathscr{F}_{\psi}=$ 
$\psi_{\sharp} \Phi_{\varphi}$, where we used $\left(\psi \varphi \psi^{-1}\right)_{\sharp}=$ id because $\psi \varphi \psi^{-1} \in \Phi_{g}$. Clearly $j$ is also $\pi_{0} \mathscr{D}$-equivariant, which means $j\left(\varphi_{*} t\right)=\varphi_{\sharp}\{j(t)\}$ for any $t \in \bigwedge^{3} H_{\mathbb{Z}} / H_{\mathbb{Z}}$. Since all $B P$ maps generate $\Phi_{g}$ by [Johnson 1979] and are conjugate to some $\varphi_{k}$ by an element of $\pi_{0} \mathscr{D}$. Thus, $\mathscr{S}$ and $-\frac{1}{2} j \circ \tau$ coincide on all BP maps. This implies $\mathscr{g}=-\frac{1}{2} j \circ \tau$ on $\mathscr{I}_{g}$.

To show Lemma 13.2, note that we can retake homomorphisms $r$ and $\mu$ for every $\varphi \in \Phi_{g}$ so as to compute $J_{\varphi}$ easily; in fact, we have shown $J_{T, \varphi}-J_{S, \varphi}=\varphi_{\sharp} v-v$ for different choices of $S$ and $T$ with common $p$ in the proofs of Theorem 2.3 and Lemma 6.5. Since $\varphi \in \Phi_{g}$ acts trivially on the cohomology group of $\Sigma_{g}$, we obtain $J_{T, \varphi}-J_{S, \varphi}=0$. In particular, the value $J_{\varphi}$ is independent of the choice of $r$ and $\mu$. So we can use $r$ defined in Section 11, namely $r\left(a_{i}^{*}\right)=\alpha_{i}$ and $r\left(b_{j}^{*}\right)=\beta_{j}$, in the computation below. With regard to $\mu$, for any $\varphi_{k}$ and $a_{0} \in \bigwedge^{2} H^{1}\left(\Sigma_{g}\right)$ we take $\mu\left(a_{0}\right)$ and extend it linearly to $\mu$ on whole $\wedge^{2} H^{1}\left(\Sigma_{g}\right)$. A connection $A$ is not needed since we compute $J$ with respect to $p=1$. The computation below is carried out using such $r$ and $\mu$.

Recall that $f_{\varphi} \in \operatorname{Hom}\left(H^{1}\left(\Sigma_{g}\right), C_{0}^{\infty}\left(\Sigma_{g}\right)\right)$ in the following lemma is the image of $\varphi=\varphi_{k}$ under the crossed homomorphism $f$ in Lemma 2.1.

Lemma 13.3. We have $f_{\varphi}\left(a_{i}^{*}\right)=0$ with $1 \leq i \leq g$, and

$$
f_{\varphi}\left(b_{j}^{*}\right)= \begin{cases}0 & \text { if } 1 \leq j \leq g, j \neq k, \\ h-h_{0} / A & \text { if } j=k .\end{cases}
$$

Proof. Since $\operatorname{supp}(\varphi) \cap \operatorname{supp}\left(\alpha_{i}\right)=\varnothing\left(\right.$ see Figure 5), we have $d f_{\varphi}\left(a_{i}^{*}\right)=\varphi_{*} \alpha_{i}-\alpha_{i}=0$. The condition $f_{\varphi}\left(a_{i}^{*}\right) \in C_{0}^{\infty}\left(\Sigma_{g}\right)$ implies $f_{\varphi}\left(a_{i}^{*}\right)=0$. For the same reason, we have $f_{\varphi}\left(b_{j}^{*}\right)=0$ for $j \neq k$. Since $d f_{\varphi}\left(b_{k}^{*}\right)=\varphi_{*} \beta_{k}-\beta_{k}, f_{\varphi}\left(b_{k}^{*}\right)$ is equal to $h$ up to a constant. The result follows from the condition $f_{\varphi}\left(b_{k}^{*}\right) \in C_{0}^{\infty}\left(\Sigma_{g}\right)$.

Next we prove Lemma 13.2 using lemmas in Section 14, which are needed only for (v) in Lemma 13.2 and are shown there.

Proof of Lemma 13.2. (i) For $a=a_{i}^{*} \wedge a_{j}^{*}$, we have $\bigwedge_{r} a=\alpha_{i} \wedge \alpha_{j}=0$ and $\kappa(a)=0$. Hence, we can take $\mu(a)=0$. Using Lemma 13.3, we have

$$
\tilde{J}_{\varphi}(a)=f_{\varphi}\left(a_{i}^{*}\right) \alpha_{j}-\varphi_{*} \alpha_{i} \cdot f_{\varphi}\left(a_{j}^{*}\right)+\varphi_{\sharp} \mu(a)-\mu(a)=0 .
$$

Similarly we obtain the equality for the other cases since all terms in $\tilde{J}_{\varphi}(a)$ are zero.

(ii) Since $\bigwedge_{r} a=0$ and $\kappa(a)=0$, we can take $\mu(a)=0$. Using Lemma 13.3, we obtain $\tilde{J}_{\varphi}(a)=-\left(h-h_{0} / A\right) \varphi_{*} \beta_{i}$. Since $\varphi=\mathrm{id}$ and $h \equiv-1$ on $\operatorname{supp} \beta_{i}$ for $i<k$, and $h \equiv 0$ for $i>k$ by Lemma 13.1, we have (ii).

(iii) and (iv) These items are shown in the same way as (i) and (ii), so we omit the proofs. 
(v) In order to compute $J_{\varphi}(a)$ for $a=a_{i}^{*} \wedge b_{i}^{*}$, we can take $\mu$ satisfying $\mu(a)=$ $\mu_{-}+\mu_{+}+\frac{1}{A} \lambda x d y-\tau_{i}$ by Lemma 14.2 and use the notation there.

Let $i \neq k$. By Lemma 13.3 we have

$$
\tilde{J}_{\varphi}(a)=\varphi_{*} \mu(a)-\mu(a)=\frac{1}{A}\left\{\varphi_{*}(\lambda x d y)-\lambda x d y\right\}-\left(\varphi_{*} \tau_{i}-\tau_{i}\right) .
$$

By Lemmas 14.3 and 14.4, we obtain, for $1 \leq l, m \leq g$,

$$
\begin{aligned}
& \int_{a_{l}} \tilde{J}_{\varphi}(a)= \begin{cases}0 & \text { if } l \neq k, \\
-\left(2 \pi^{2}+A_{+}\right) / A & \text { if } l=k, i<k, \\
-\left(2 \pi^{2}-A_{-}\right) / A & \text { if } l=k, i>k,\end{cases} \\
& \int_{b_{m}} \tilde{J}_{\varphi}(a)=0 .
\end{aligned}
$$

This case follows from $A_{+}+A_{-}=A, h_{0}=2 \pi^{2}-A_{-}$(which is Lemma 14.5) and the fact that $\tilde{J}_{\varphi}(a)$ is a closed form.

Let $i=k$. We have

$$
\tilde{J}_{\varphi}(a)=-\varphi_{*} \alpha_{k} \cdot f_{\varphi}\left(b_{k}^{*}\right)+\varphi_{*} \mu(a)-\mu(a)=\frac{h_{0}}{A} \alpha_{k}+\varphi_{*} \mu(a)-\mu(a),
$$

where we use $\varphi=\mathrm{id}$ and $h \equiv 0$ on $\operatorname{supp} \alpha_{k}$. By a similar computation of $\int_{c} \tilde{J}_{\varphi}(a)$ with $c=a_{l}, b_{m}$ as above, we obtain

$$
J_{\varphi}(a)=\frac{h_{0}}{A} a_{k}^{*}+\frac{-2 \pi^{2}-\left(-A_{-}\right)}{A} a_{k}^{*}=0
$$

by Lemma 14.5. This implies (v).

\section{The main part of the computation}

In this section we will show the lemmas needed to prove Lemma 13.2. These are the main parts of the computation of $J_{\varphi}(a)$. Throughout this section, we fix the integer $k$ as $1<k<g$.

Let $\lambda: \Sigma_{g} \rightarrow[0,1]$ be a smooth function with support as depicted in Figure 6 such that

$$
\left\{\begin{array}{l}
\operatorname{supp} \lambda \subset T_{k} \\
\partial(\operatorname{supp} \lambda) \cong \amalg^{3} S^{1} \\
\operatorname{supp} d \lambda \subset \operatorname{small} \text { neighborhood of } \partial(\operatorname{supp} \lambda) \\
\lambda \equiv 1 \text { on supp } \lambda \backslash \operatorname{supp} d \lambda .
\end{array}\right.
$$

Since $\varepsilon>0$ is sufficiently small, we can also assume

$$
T_{k}^{\prime}:=T_{k} \cap\{(x, y) \mid-4 \varepsilon \leq x \leq 0\} \subset \lambda^{-1}(1) .
$$




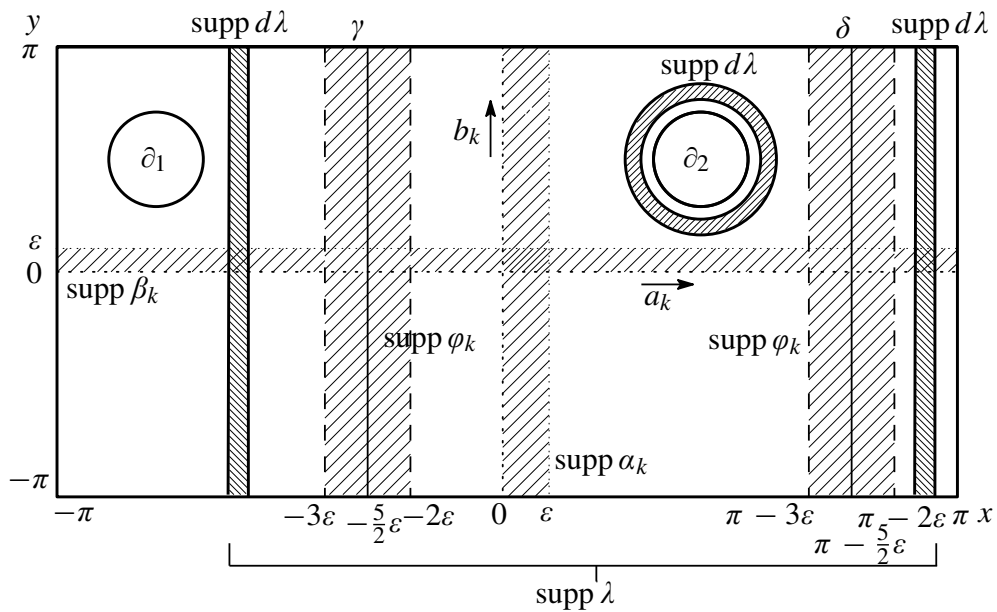

Figure 6. Support of $\lambda$.

Let $\lambda_{ \pm}: \Sigma_{g} \rightarrow[0,1]$ be two smooth functions uniquely defined by

$$
\left\{\begin{array}{l}
\lambda_{-}+\lambda_{+} \lambda_{+}=1, \\
\operatorname{supp} \lambda_{-} \subset \Sigma_{k-} \cup T_{k}, \\
\operatorname{supp} \lambda_{+} \subset \Sigma_{k+} \cup T_{k}, \\
\operatorname{supp} \lambda_{-} \cap \operatorname{supp} \lambda_{+}=\varnothing .
\end{array}\right.
$$

As depicted in Figure 6, supp $\lambda_{+}$and supp $\lambda_{-}$are closed subsurfaces of $\Sigma_{g}$ with one circle boundary and two respectively.

Hereafter, we assume $\left.\omega\right|_{T_{k}}=d x \wedge d y$ since the crossed homomorphism $\mathscr{F}$ is independent of the choice of $\omega$.

We consider the 2 -form $d(\lambda x d y)$ on $T_{k}$ as that on $\Sigma_{g}$ by extending it by 0 on $\Sigma_{g} \backslash T_{k}$. Let $\omega_{ \pm} \in \Omega^{2}\left(\Sigma_{g}\right)$ be the two closed 2-forms defined by supp $\omega_{ \pm} \subset \operatorname{supp} \lambda_{ \pm}$ and

$$
\omega_{-}+d(\lambda x d y)+\omega_{+}=\omega .
$$

Set $A_{ \pm}=\int_{\Sigma_{g}} \omega_{ \pm}$. We have $A_{-}+A_{+}=A$.

Lemma 14.1. For any $i$ with $1 \leq i \leq g$ and $p, q \in \mathbb{R}$ with $p+q=1$, there exists $\tau_{i} \in \Omega^{1}\left(\Sigma_{g}\right)$ satisfying

(i) $\alpha_{i} \wedge \beta_{i}=p \alpha_{1} \wedge \beta_{1}+q \alpha_{g} \wedge \beta_{g}+d \tau_{i}$,

(ii) $\operatorname{supp} \tau_{i} \subset$ the image of an embedding of a rectangle,

(iii) $\operatorname{supp} \tau_{i} \cap \operatorname{supp} \varphi \subset T_{k}^{\prime}$, and

(iv) $\left.\tau_{i}\right|_{T_{k}^{\prime}}=s d \rho(y)$ with $s= \begin{cases}q & \text { if } i<k, \\ -p & \text { if } i \geqq k .\end{cases}$ 


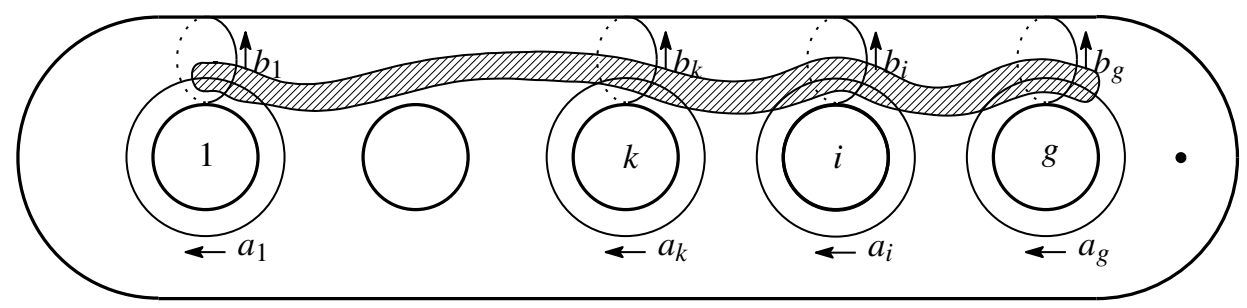

Figure 7. Support of $\tau_{i}$.

Proof. Let $\rho$ be the smooth function on $\mathbb{R}$ in Section 11. For any $t_{-}, t_{0}, t_{+} \in \mathbb{R}$ satisfying $t_{-}+\varepsilon \leq t_{0}+\varepsilon \leq t_{+}$, we define the smooth function $\rho_{W}: \mathbb{R} \rightarrow[0,1]$, where $W=\left\{t_{-}, t_{0}, t_{+}, p, q\right\}$, by

$$
\rho_{W}(t)= \begin{cases}0 & \text { if } t<t_{-}, \\ -p \rho\left(t-t_{0}\right) & \text { if } t_{-} \leq t<t_{-}+\varepsilon, \\ -p & \text { if } t_{1}+\varepsilon \leq t<t_{0} \\ -p+\rho\left(t-t_{0},\right. & \text { if } t_{0} \leq t<t_{0}+\varepsilon, \\ 1-p(=q) & \text { if } t_{0}+\varepsilon \leq t<t_{+}, \\ q\left\{1-\rho\left(t-t_{+}\right)\right\} & \text {if } t_{+} \leq t<t_{+}+\varepsilon, \\ 0 & \text { if } t_{+}+\varepsilon \leq t\end{cases}
$$

for $t \in \mathbb{R}$. Let $\tilde{\tau}_{W} \in \Omega^{1}(\mathbb{R} \times[-\varepsilon, 2 \varepsilon])$ be the 1 -form defined by $\tilde{\tau}_{W}(x, y)=$ $\rho_{W}(x) d \rho(y)$ for $(x, y) \in \mathbb{R} \times[-\varepsilon, 2 \varepsilon]$. We have

$$
d \tilde{\tau}_{W}(x, y)= \begin{cases}0 & \text { if } x<t_{-}, \\ -p d \rho\left(x-t_{0}\right) \wedge d \rho(y) & \text { if } t_{-} \leq x<t_{-}+\varepsilon, \\ 0 & \text { if } t_{-}+\varepsilon \leq x<t_{0}, \\ d \rho\left(x-t_{0}\right) \wedge d \rho(y) & \text { if } t_{0} \leq x<t_{0}+\varepsilon, \\ 0 & \text { if } t_{0}+\varepsilon \leq x<t_{+}, \\ -q d \rho\left(x-t_{+}\right) \wedge d \rho(y) & \text { if } t_{+} \leq x<t_{+}+\varepsilon, \\ 0 & \text { if } t_{+}+\varepsilon \leq x\end{cases}
$$

By the definition of $\alpha_{i}$ and $\beta_{i}$ in Section 11, we have $\alpha_{i} \wedge \beta_{i}=d \rho(x) \wedge d \rho(y)$ on the local coordinates $(x, y) \in T_{i}$ for each $1 \leq i \leq g$. So, we can appropriately choose $t_{+}, t_{0}, t_{-} \in \mathbb{R}$ and an embedding $\left[t_{-}, t_{+}+\varepsilon\right] \times[-\varepsilon, 2 \varepsilon] \hookrightarrow \Sigma_{g}$ as depicted in Figure 7 such that the extension $\tau_{i}$ of $\tilde{\tau}_{W}$ by 0 on the complement of the image of the embedding satisfies the required properties.

We apply Lemma 14.1 for $p=A_{-} / A$ and $q=A_{+} / A$, we then have $\tau_{i} \in \Omega^{1}\left(\Sigma_{g}\right)$. Let $a=a_{i}^{*} \wedge b_{i}^{*}$; then $\kappa(a)=1 / A$. Using (14-1) and (i) in Lemma 14.1, we obtain

$$
\kappa(a) \omega-\alpha_{i} \wedge \beta_{i}=\frac{1}{A} \omega_{-}-p \alpha_{1} \wedge \beta_{1}+\frac{1}{A} \omega_{+}-q \alpha_{g} \wedge \beta_{g}+d\left\{\frac{1}{A} \lambda x d y-\tau_{i}\right\} .
$$


Since

$$
\operatorname{supp}\left(\frac{1}{A} \omega_{-}-p \alpha_{1} \wedge \beta_{1}\right) \subset \operatorname{supp} \lambda_{-}, \quad \operatorname{supp}\left(\frac{1}{A} \omega_{+}-q \alpha_{g} \wedge \beta_{g}\right) \subset \operatorname{supp} \lambda_{+},
$$

and

$$
\int_{\operatorname{supp} \lambda_{-}}\left(\frac{1}{A} \omega_{-}-p \alpha_{1} \wedge \beta_{1}\right)=\int_{\operatorname{supp} \lambda_{+}}\left(\frac{1}{A} \omega_{+}-q \alpha_{g} \wedge \beta_{g}\right)=0,
$$

there exist $\mu_{-}, \mu_{+} \in \Omega^{1}\left(\Sigma_{g}\right)$ such that $\operatorname{supp} \mu_{-} \subset \operatorname{supp} \lambda_{-}, \operatorname{supp} \mu_{+} \subset \lambda_{+}, d \mu_{-}=$ $\frac{1}{A} \omega_{-}-p \alpha_{1} \wedge \beta_{1}$ and $d \mu_{+}=\frac{1}{A} \omega_{+}-q \alpha_{g} \wedge \beta_{g}$. Thus, we have

$$
\kappa(a) \omega-\alpha_{i} \wedge \beta_{i}=d\left(\mu_{-}+\mu_{+}+\frac{1}{A} \lambda x d y-\tau_{i}\right) .
$$

Hence, we can take

$$
\mu(a)=\mu_{-}+\mu_{+}+\frac{1}{A} \lambda x d y-\tau_{i}
$$

Thus, we have:

Lemma 14.2. In the situation above, for every $\varphi=\varphi_{k}$ and $a=a_{i}^{*} \wedge b_{i}^{*}$, there exists a homomorphism $\mu: \wedge^{2} H^{1}\left(\Sigma_{g}\right) \rightarrow \Omega^{1}\left(\Sigma_{g}\right)$ in Lemma 2.2 satisfying (14-2).

Lemma 14.3. (i) $\int_{a_{j}}\left\{\varphi_{*}(\lambda x d y)-\lambda x d y\right\}= \begin{cases}0 & \text { if } j \neq k, \\ -2 \pi^{2} & \text { if } j=k,\end{cases}$

(ii) $\int_{b_{j}}\left\{\varphi_{*}(\lambda x d y)-\lambda x d y\right\}=0$ for all $j$.

Proof. Since $\operatorname{supp}\left\{\varphi_{*}(\lambda x d y)-\lambda x d y\right\} \subset \operatorname{supp} \varphi$ is disjoint from $a_{j}(j \neq k)$ and $b_{j}$ for all $j$, the integrals along them are equal to 0 . So, we have only to compute the integral along $a_{k}$. We recall that $\lambda$ is equal to 1 on $\operatorname{supp} \varphi$. On $T_{k}$, we have

$$
\begin{aligned}
\varphi_{*}(\lambda x d y)-\lambda x d y & =\left(\varphi^{-1}\right)^{*}(x d y)-x d y \\
& = \begin{cases}-x a^{\prime}(x) d x & (x, y) \in[-3 \varepsilon,-2 \varepsilon] \times(-\pi, \pi] \\
x a^{\prime}(x-\pi) d x & (x, y) \in[\pi-3 \varepsilon, \pi-2 \varepsilon] \times(-\pi, \pi] \\
0 & \text { otherwise; }\end{cases}
\end{aligned}
$$

hence,

$$
\begin{aligned}
\int_{a_{k}}\left\{\varphi_{*}(\lambda x d y)-\lambda x d y\right\} & =\int_{-3 \varepsilon}^{-2 \varepsilon}\left\{-x a^{\prime}(x)\right\} d x+\int_{\pi-3 \varepsilon}^{\pi-2 \varepsilon} x a^{\prime}(x-\pi) d x \\
& =\pi\{a(-2 \varepsilon)-a(-3 \varepsilon)\}=-2 \pi^{2} .
\end{aligned}
$$
Lemma 14.4. (i) $\int_{a_{j}}\left(\varphi_{*} \tau_{i}-\tau_{i}\right)= \begin{cases}0 & \text { if } j \neq k, \\ q=A_{+} / A & \text { if } j=k, i<k, \\ -p=-A_{-} / A & \text { if } j=k, i \geqq k\end{cases}$
Lemma 14.4. (i) $\int_{a_{j}}\left(\varphi_{*} \tau_{i}-\tau_{i}\right)= \begin{cases}0 & \text { if } j \neq k, \\ q=A_{+} / A & \text { if } j=k, i<k, \\ -p=-A_{-} / A & \text { if } j=k, i \geqq k\end{cases}$
Lemma 14.4. (i) $\int_{a_{j}}\left(\varphi_{*} \tau_{i}-\tau_{i}\right)= \begin{cases}0 & \text { if } j \neq k, \\ q=A_{+} / A & \text { if } j=k, i<k, \\ -p=-A_{-} / A & \text { if } j=k, i \geqq k\end{cases}$

(ii) $\int_{b_{j}}\left(\varphi_{*} \tau_{i}-\tau_{i}\right)=0$ for all $j$. 
Proof. We have only to compute the integral along $a_{k}$ since the others are clear by considering the support of the integrand. Let $\tilde{\rho}$ be the smooth function on $\mathbb{R}$ with $\tilde{\rho}(0)=0$ whose differential $d \tilde{\rho}$ agrees with the pullback of $d \rho$ by the projection $\mathbb{R} \rightarrow \mathbb{R} / 2 \pi \mathbb{Z}$. By Lemma 14.1 , on $T_{k}^{\prime}$ we have

$$
\begin{aligned}
\varphi_{*} \tau_{i}-\tau_{i} & =\left(\varphi^{-1}\right)^{*}\{s d \rho(y)\}-s d \rho(y) \\
& =-s \tilde{\rho}^{\prime}(-a(x)+y) a^{\prime}(x) d x+s\left\{\tilde{\rho}^{\prime}(-a(x)+y)-\tilde{\rho}^{\prime}(y)\right\} d y
\end{aligned}
$$

for $(x, y) \in[-3 \varepsilon,-2 \varepsilon] \times(-\pi, \pi]$, and $\varphi_{*} \tau_{i}-\tau_{i}=0$ otherwise. Since

$$
\operatorname{supp}\left(\varphi_{*} \tau_{i}-\tau_{i}\right) \subset T_{k}^{\prime},
$$

we have

$$
\int_{a_{k}}\left(\varphi_{*} \tau_{i}-\tau_{i}\right)=-s \int_{-3 \varepsilon}^{-2 \varepsilon} \tilde{\rho}^{\prime}(-a(x)+0) a^{\prime}(x) d x=s[\tilde{\rho}(-a(x))]_{-3 \varepsilon}^{-2 \varepsilon}=s .
$$

Finally, we prove the following lemma:

$$
\text { Lemma 14.5. } h_{0}=2 \pi^{2}-A_{-} \text {. }
$$

Proof. Since supp $h \cap \operatorname{supp} \lambda_{+}=\varnothing$ and $h \equiv-1$ on supp $\lambda_{-}$, we have

$$
h=\left(\lambda_{-}+\lambda+\lambda_{+}\right) h=-\lambda_{-}+\lambda h
$$

and

$$
h_{0}=\int_{\Sigma_{g}} h \omega=-\int_{\Sigma_{g}} \lambda_{-} \omega+\int_{\Sigma_{g}} \lambda h \omega .
$$

Since $d h=\varphi_{*} \beta_{k}-\beta_{k}$ by definition and $\left.\omega\right|_{T_{k}}=d x \wedge d y$ by assumption, we obtain

$$
d(\lambda h x d y)=d \lambda \wedge h x d y+\lambda\left(\varphi_{*} \beta_{k}-\beta_{k}\right) \wedge x d y+\lambda h \omega .
$$

Then we have

$$
\int_{\Sigma_{g}} \lambda h \omega=-\int_{\Sigma_{g}} d \lambda_{-} \wedge x d y-\int_{\Sigma_{g}}\left(\varphi_{*} \beta_{k}-\beta_{k}\right) \wedge x d y
$$

by Stokes' formula and $h \equiv-1$ on $\operatorname{supp} d \lambda \cap \operatorname{supp} h \subset \operatorname{supp} d \lambda_{-}$.

On the other hand, since supp $\lambda \subset T_{k}$, we have

$$
\lambda \omega=d(\lambda x d y)-d \lambda \wedge x d y=d \lambda_{-} \wedge x d y+d(\lambda x d y)+d \lambda_{+} \wedge x d y
$$

and

$$
\omega=\lambda_{-} \omega+d \lambda_{-} \wedge x d y+d(\lambda x d y)+d \lambda_{+} \wedge x d y+\lambda_{+} \omega .
$$

By considering supports, we get $\omega_{-}=\lambda_{-} \omega+d \lambda_{-} \wedge x d y$ and

$$
-\int_{\Sigma_{g}} \lambda_{-} \omega=-\int_{\Sigma_{g}} \omega_{-}+\int_{\Sigma_{g}} d \lambda_{-} \wedge x d y .
$$


Summing up equalities (14-3), (14-4), and (14-5), we have

$$
h_{0}=-\int_{\Sigma_{g}} \omega_{-}-\int_{\Sigma_{g}}\left(\varphi_{*} \beta_{k}-\beta_{k}\right) \wedge x d y .
$$

Since $\beta_{k}=d \rho(y)=d \tilde{\rho}(y)$ on $T_{k}$, we have

$$
\begin{aligned}
& \left(\varphi_{*} \beta_{k}-\beta_{k}\right) \wedge x d y=d\left[\varphi_{*}\{\rho \tilde{(y)}\}\right] \wedge x d y= \\
& \begin{cases}-\tilde{\rho}^{\prime}(-a(x)+y) a^{\prime}(x) d x \wedge x d y & \text { if }(x, y) \in[-3 \varepsilon,-2 \varepsilon] \times(-\pi, \pi], \\
\tilde{\rho}^{\prime}(a(x-\pi)+y) a^{\prime}(x-\pi) d x \wedge x d y & \text { if }(x, y) \in[\pi-3 \varepsilon, \pi-2 \varepsilon] \times(-\pi, \pi], \\
0 & \text { otherwise, }\end{cases}
\end{aligned}
$$

and then we obtain

$$
\begin{aligned}
\int_{\Sigma_{g}}\left(\varphi_{*} \beta_{k}-\beta_{k}\right) \wedge x d y & =-\int_{-3 \varepsilon}^{-2 \varepsilon} a^{\prime}(x) x d x+\int_{\pi-3 \varepsilon}^{\pi-2 \varepsilon} a^{\prime}(x-\pi) x d x \\
& =\pi \int_{-3 \varepsilon}^{-2 \varepsilon} a^{\prime}(x) d x=\pi[a(x)]_{-3 \varepsilon}^{-2 \varepsilon}=-2 \pi^{2} .
\end{aligned}
$$

Since $\int_{\Sigma_{g}} \omega_{-}=A_{-}$, we have $h_{0}=-A_{-}+2 \pi^{2}$.

\section{References}

[Banyaga 1997] A. Banyaga, The structure of classical diffeomorphism groups, Mathematics and its Applications 400, Kluwer, Dordrecht, 1997. MR 98h:22024 Zbl 0874.58005

[Brown 1982] K. S. Brown, Cohomology of groups, Graduate Texts in Mathematics 87, Springer, New York, 1982. MR 83k:20002 Zbl 0584.20036

[Day 2007] M. B. Day, "Extending Johnson's and Morita's homomorphisms to the mapping class group”, Algebr. Geom. Topol. 7 (2007), 1297-1326. MR 2008j:57033 Zbl 1181.57025

[Day 2011] M. B. Day, "Extended flux maps on surfaces and the contracted Johnson homomorphism", J. Symplectic Geom. 9:4 (2011), 445-482. MR 2900787 Zbl 06049656

[Hatcher 1978] A. E. Hatcher, "Concordance spaces, higher simple-homotopy theory, and applications”, pp. 3-21 in Algebraic and geometric topology, I (Stanford, CA, 1976), edited by R. J. Milgram, Proc. Sympos. Pure Math. 32, Amer. Math. Soc., Providence, R.I., 1978. MR 80f:57014 Zbl 0406.57031

[Hsiang and Sharpe 1976] W. C. Hsiang and R. W. Sharpe, "Parametrized surgery and isotopy", Pacific J. Math. 67:2 (1976), 401-459. MR 58 \#13091 Zbl 0348.57015

[Johnson 1979] D. L. Johnson, "Homeomorphisms of a surface which act trivially on homology", Proc. Amer. Math. Soc. 75:1 (1979), 119-125. MR 80h:57008 Zbl 0407.57003

[Johnson 1980] D. Johnson, "An abelian quotient of the mapping class group $\Phi_{g}$ ", Math. Ann. 249:3 (1980), 225-242. MR 82a:57008 Zbl 0409.57009

[Kasagawa 2008] R. Kasagawa, "On crossed homomorphisms on symplectic mapping class groups", Trans. Amer. Math. Soc. 360:9 (2008), 4815-4839. MR 2009f:57040 Zbl 1158.53056

[Kędra et al. 2006] J. Kędra, D. Kotschick, and S. Morita, "Crossed flux homomorphisms and vanishing theorems for flux groups”, Geom. Funct. Anal. 16:6 (2006), 1246-1273. MR 2008c:53091 Zbl 1114.57031 
[Kotschick and Morita 2005] D. Kotschick and S. Morita, "Signatures of foliated surface bundles and the symplectomorphism groups of surfaces", Topology 44:1 (2005), 131-149. MR 2005k:57050 Zbl 1163.57304

[Kotschick and Morita 2007] D. Kotschick and S. Morita, "Characteristic classes of foliated surface bundles with area-preserving holonomy”, J. Diff. Geom. 75:2 (2007), 273-302. MR 2008k:57046 Zbl 1115.57016

[McDuff 1983] D. McDuff, "Some canonical cohomology classes on groups of volume preserving diffeomorphisms", Trans. Amer. Math. Soc. 275:1 (1983), 345-356. MR 84h:58154 Zbl 0522.57029

[Milnor 2011] J. Milnor, "Differential topology forty-six years later", Notices Amer. Math. Soc. 58:6 (2011), 804-809. MR 2012i:57001 Zbl 1225.01040

[Morita 1993] S. Morita, "The extension of Johnson's homomorphism from the Torelli group to the mapping class group”, Invent. Math. 111:1 (1993), 197-224. MR 93j:57001 Zbl 0787.57008

[Morita and Penner 2008] S. Morita and R. C. Penner, "Torelli groups, extended Johnson homomorphisms, and new cycles on the moduli space of curves", Math. Proc. Cambridge Philos. Soc. 144:3 (2008), 651-671. MR 2009c:32028 Zbl 1157.32015

[Moser 1965] J. Moser, "On the volume elements on a manifold", Trans. Amer. Math. Soc. 120 (1965), 286-294. MR 32 \#409 Zbl 0141.19407

[Perron 2004] B. Perron, "Homomorphic extensions of Johnson homomorphisms via Fox calculus", Ann. Inst. Fourier (Grenoble) 54:4 (2004), 1073-1106. MR 2005k:57034 Zbl 1109.57013

[Vaisman 1987] I. Vaisman, Symplectic geometry and secondary characteristic classes, Progress in Mathematics 72, Birkhäuser, Boston, MA, 1987. MR 89f:58062 Zbl 0629.53002

Received June 7, 2012. Revised January 22, 2013.

RYOJI KASAGAWA

Department of Mathematics, College of Science and Technology

NIHON UNIVERSITY

1-8 KANDA, SURUGAdAI, CHIYOdA-KU

TOKYO 101-8308

JAPAN

kasagawa@math.cst.nihon-u.ac.jp 


\title{
PACIFIC JOURNAL OF MATHEMATICS
}

\author{
msp.org/pjm
}

Founded in 1951 by E. F. Beckenbach (1906-1982) and F. Wolf (1904-1989)

\section{EDITORS}

V. S. Varadarajan (Managing Editor)

Department of Mathematics

University of California

Los Angeles, CA 90095-1555

pacific@math.ucla.edu

Paul Balmer

Department of Mathematics

University of California

Los Angeles, CA 90095-1555

balmer@math.ucla.edu

Daryl Cooper

Department of Mathematics

University of California

Santa Barbara, CA 93106-3080 cooper@math.ucsb.edu

Jiang-Hua $\mathrm{Lu}$

Department of Mathematics

Pokfulam Rd., Hong Kong jhlu@maths.hku.hk
The University of Hong Kong

Don Blasius

Department of Mathematics University of California

Los Angeles, CA 90095-1555

blasius@math.ucla.edu

Robert Finn

Department of Mathematics Stanford University

Stanford, CA 94305-2125

finn@math.stanford.edu

Sorin Popa

Department of Mathematics

University of California

Los Angeles, CA 90095-1555 popa@math.ucla.edu

Paul Yang

Department of Mathematics Princeton University

Princeton NJ 08544-1000

yang@math.princeton.edu

\section{PRODUCTION}

Silvio Levy, Scientific Editor, production@msp.org

\section{SUPPORTING INSTITUTIONS}

ACADEMIA SINICA, TAIPEI

CALIFORNIA INST. OF TECHNOLOGY

INST. DE MATEMÁTICA PURA E APLICADA

KEIO UNIVERSITY

MATH. SCIENCES RESEARCH INSTITUTE

NEW MEXICO STATE UNIV.

OREGON STATE UNIV.

\author{
STANFORD UNIVERSITY \\ UNIV. OF BRITISH COLUMBIA \\ UNIV. OF CALIFORNIA, BERKELEY \\ UNIV. OF CALIFORNIA, DAVIS \\ UNIV. OF CALIFORNIA, LOS ANGELES \\ UNIV. OF CALIFORNIA, RIVERSIDE \\ UNIV. OF CALIFORNIA, SAN DIEGO \\ UNIV. OF CALIF., SANTA BARBARA
}

\author{
Vyjayanthi Chari \\ Department of Mathematics \\ University of California \\ Riverside, CA 92521-0135 \\ chari@math.ucr.edu \\ Kefeng Liu \\ Department of Mathematics \\ University of California \\ Los Angeles, CA 90095-1555 \\ liu@math.ucla.edu \\ Jie Qing \\ Department of Mathematics \\ University of California \\ Santa Cruz, CA 95064 \\ qing@cats.ucsc.edu
}

These supporting institutions contribute to the cost of publication of this Journal, but they are not owners or publishers and have no responsibility for its contents or policies.

See inside back cover or msp.org/pjm for submission instructions.

The subscription price for 2013 is US \$400/year for the electronic version, and \$485/year for print and electronic.

Subscriptions, requests for back issues and changes of subscribers address should be sent to Pacific Journal of Mathematics, P.O. Box 4163, Berkeley, CA 94704-0163, U.S.A. The Pacific Journal of Mathematics is indexed by Mathematical Reviews, Zentralblatt MATH, PASCAL CNRS Index, Referativnyi Zhurnal, Current Mathematical Publications and the Science Citation Index.

The Pacific Journal of Mathematics (ISSN 0030-8730) at the University of California, c/o Department of Mathematics, 798 Evans Hall \#3840, Berkeley, CA 94720-3840, is published twelve times a year. Periodical rate postage paid at Berkeley, CA 94704, and additional mailing offices. POSTMASTER: send address changes to Pacific Journal of Mathematics, P.O. Box 4163, Berkeley, CA 94704-0163.

PJM peer review and production are managed by EditFLOW ${ }^{\circledR}$ from Mathematical Sciences Publishers.

\section{PUBLISHED BY}

mathematical sciences publishers

nonprofit scientific publishing

http://msp.org/

(C) 2013 Mathematical Sciences Publishers 


\section{PACIFIC JOURNAL OF MATHEMATICS}

Volume $265 \quad$ No. $2 \quad$ October 2013

Singularity removability at branch points for Willmore surfaces

YANN BERNARD and TRISTAN RIVIÈRE

On Bach flat warped product Einstein manifolds

QIANG CHEN and CHENXU HE

On plane sextics with double singular points

Alex DeGTyareV

A computational approach to the Kostant-Sekiguchi correspondence

HEIKo DiETRICH and WILLEM A. DE GRAAF

Landau-Toeplitz theorems for slice regular functions over quaternions

GRAZIANO GENTILI and GiUlia SARFATTI

On surgery curves for genus-one slice knots

PATRICK M. GILMER and CHARLES LIVINGSTON

Characterizing abelian varieties by the reduction of the Mordell-Weil group

Chris Hall and Antonella Perucca

Variation of complex structures and the stability of Kähler-Ricci

Solitons

StUART J. HALl and ThOMAS MURPHY

On crossed homomorphisms of the volume preserving diffeomorphism groups

RYOJI KASAGAWA

Regularity at the boundary and tangential regularity of solutions of the Cauchy-Riemann system

TRAN VU KHANH and GIUSEPPE ZAMPIERI

On the Steinberg character of a semisimple $p$-adic group 